\title{
New approach to solutions of a class of singular fractional $q$-differential problem via quantum calculus
}

\author{
Sihua Liang ${ }^{1}$ and Mohammad Esmael Samei ${ }^{2^{*}}$ (D)
}

\author{
"Correspondence: \\ mesamei@gmail.com; \\ mesamei@basu.ac.ir \\ ${ }^{2}$ Department of Mathematics, Bu-Ali \\ Sina University, Hamedan, Iran \\ Full list of author information is \\ available at the end of the article
}

\begin{abstract}
In the present article, by using the fixed point technique and the Arzelà-Ascoli theorem on cones, we wish to investigate the existence of solutions for a non-linear problems regular and singular fractional $q$-differential equation

$$
\left({ }^{c} D_{q}^{\alpha} f\right)(t)=w\left(t, f(t), f^{\prime}(t),\left({ }^{c} D_{q}^{\beta} f\right)(t)\right),
$$

under the conditions $f(0)=c_{1} f(1), f^{\prime}(0)=c_{2}\left({ }^{c} D_{q}^{\beta} f\right)(1)$ and

$f^{\prime \prime}(0)=f^{\prime \prime \prime}(0)=\cdots=f^{(n-1)}(0)=0$, where $\alpha \in(n-1, n)$ with $n \geq 3, \beta, q \in J=(0,1), c_{1} \in J$,

$c_{2} \in\left(0, \Gamma_{q}(2-\beta)\right)$, the function $w$ is $L^{k}$-Carathéodory, $w\left(t, x_{1}, x_{2}, x_{3}\right)$ and may be

singular and ${ }^{C} D_{q}^{\alpha}$ the fractional Caputo type $q$-derivative. Of course, here we applied the definitions of the fractional $q$-derivative of Riemann-Liouville and Caputo type by presenting some examples with tables and algorithms; we will illustrate our results, too.
\end{abstract}

MSC: Primary 34A08; 34B16; secondary 39A13

Keywords: Singularity; Caputo q-derivative; Quantum calculus; q-differential

\section{Introduction}

The fractional calculus and $q$-calculus deal with the generalization of integration and differentiation of integer order to any order. It is known that fractional calculus is used for a better description of phenomena having both discrete and continuous behaviors, and applying in different sciences and engineering such as mechanics, electricity, biology, control theory, signal and image processing [1-12]. It has an old history and several fractional derivations where defined, such as the Caputo, the Riemann-Liouville and the Caputo and Fabrizio derivations. These derivations appeared recently in much work on integrodifferential equations by using different views which young researchers could use for their work [13-27]. The fractional $q$-calculus has been applied to almost very field of non-linear mathematics analysis [28-38]. This branch of mathematics was introduced by Jackson in 1910 [1, 39]. For earlier work on the topic, we refer to [40, 41], whereas the preliminary concepts on $q$-fractional calculus can be found in [4]. For some applications of the

(c) The Author(s) 2020. This article is licensed under a Creative Commons Attribution 4.0 International License, which permits use, sharing, adaptation, distribution and reproduction in any medium or format, as long as you give appropriate credit to the original author(s) and the source, provide a link to the Creative Commons licence, and indicate if changes were made. The images or other third party material in this article are included in the article's Creative Commons licence, unless indicated otherwise in a credit line to the material. If material is not included in the article's Creative Commons licence and your intended use is not permitted by statutory regulation or exceeds the permitted use, you will need to obtain permission directly from the copyright holder. To view a copy of this licence, visit http://creativecommons.org/licenses/by/4.0/. 
$q$-fractional calculus, see for example [2, 3, 5, 7, 8, 42-44]. Also, there has been a significant increase in knowledge in the field of differential and $q$-differential equations and inclusions in recent years [45-49].

In 2012, Ahmad et al., studied the existence and uniqueness of solutions for the fractional $q$-difference equations ${ }^{c} D_{q}^{\alpha} u(t)=T(t, u(t))$ with the boundary conditions $\alpha_{1} u(0)$ $\beta_{1} D_{q} u(0)=\gamma_{1} u\left(\eta_{1}\right)$ and $\alpha_{2} u(1)-\beta_{2} D_{q} u(1)=\gamma_{2} u\left(\eta_{2}\right)$, where $\alpha \in(1,2], \alpha_{i}, \beta_{i}, \gamma_{i}, \eta_{i}$ are real numbers, for $i=1,2$ and $T \in C(J \times \mathbb{R}, \mathbb{R})$ [34]. In 2013, Baleanu et al., reviewed the nonlinear singular fractional problem $\left({ }^{c} D^{\alpha} u\right)(t)=w\left(t, u(t), u^{\prime}(t),\left({ }^{c} D^{\beta} u\right)(t)\right)$, under the boundary conditions $u(0)=a_{1} u(1), u^{\prime}(0)=a_{2}\left({ }^{c} D^{\beta} u\right)(1)$ and $u^{\prime \prime}(0)=u^{\prime \prime \prime}(0)=\cdots=u^{(n-1)}(0)=0$ on cones, where $\alpha \in(n-1, n)$ with an integer number $n \geq 3, \beta, a_{1}, a_{2} \in J=(0,1),(-\infty, 1)$, $(0, \Gamma(2-\beta))$, respectively, and $w$ is a $L^{\kappa}$-Carathéodory function, $\kappa(\alpha-1)>1$, with the same conditions, which is was addressed by Agarwal et al. [50]. In 2013, Zhao el al. [38] reviewed the $q$-integral problem $\left(D_{q}^{\alpha} u\right)(t)+f(t, u(t))=0$, with the conditions $u(1)$, $u(0)$ equal to $\mu I_{q}^{\beta} u(\eta), 0$, respectively, for almost all $t \in(0,1)$, where $q \in(0,1)$ and $\alpha$, $\beta, \eta$ belong to $(1,2],(0,2],(0,1)$, respectively, $\mu$ is positive real number, $D_{q}^{\alpha}$ is the $q$ derivative of Riemann-Liouville and real-values continuous map $u$ defined on $I \times[0, \infty)$. In 2014, Jiang et al., investigated the existence and uniqueness of solution of the problem $D_{q}^{\beta}\left(\phi_{p}\left(D_{q}^{\alpha} y(x)\right)\right)+w\left(x, y(x), D_{q}^{\gamma} y(x)\right)=0$, under the conditions $y(0)=D_{q} y(0)=D_{q}^{\alpha} y(0)=0$ and $y(1)=\mu I_{q} y(\eta)$, by invoking the $\mathrm{p}$-Laplacian operator, where $w$ belongs to $C(E, \mathbb{R})$ with $E=[0,1] \times \mathbb{R}^{2}, \alpha$ and $\beta, q, \eta, \gamma$ belong to in $(2,3)$ and $(0,1)$, respectively, $\mu>0$ is constant, $D_{q}^{\alpha}$ is the fractional $q$-derivative of the Riemann-Liouville type, $D_{q}$ and $I_{q}$ denote the $q$ derivative and the $q$-integral, receptively, and $\phi_{p}$ is the p-Laplacian operator defined by $\phi_{p}(s)=|s|^{p-2} s$, with $p>1[51]$.

Two year later, in 2016, Abdeljawad et al. [52] stated and proved a new discrete $q$ fractional version of the Gronwall inequality: $\left.{ }_{q} C_{a}^{\alpha} f\right)(t)=w(t, f(t))$ and $f(a)=\gamma$ such that $\alpha \in(0,1], a \in \mathbb{T}_{q}=\left\{q^{n}: n \in \mathbb{Z}\right\}$, t belongs to $\mathbb{T}_{a}=[0, \infty)_{q}=\left\{q^{-i} a: i=0,1,2, \ldots\right\},{ }_{q} C_{a}^{\alpha}$ means the Caputo fractional difference of order $\alpha$ and $w(t, x)$ fulfills a Lipschitz condition for all $t$ and $x$. Then, in 2017, Zhou et al. [53] provided existence criteria for the solutions of the fractional Langevin differential equation under some conditions:

$$
\left\{\begin{array}{l}
D_{0^{+}}^{\beta} \phi_{p}\left[\left(D_{0^{+}}^{\alpha}+\eta\right) f(t)\right]=w\left(t, f(t), D_{0^{+}}^{\alpha} f(t)\right), \\
f(0)=-f(1), \quad D_{0^{+}}^{\alpha} f(0)=-D_{0^{+}}^{\alpha} f(1),
\end{array}\right.
$$

and

$$
\left\{\begin{array}{l}
{ }_{q} D_{0^{+}}^{\beta} \phi_{p}\left[\left(D_{0^{+}}^{\alpha}+\eta\right) f(t)\right]=w\left(t, f(t),{ }_{q} D_{0^{+}}^{\alpha} f(t)\right), \\
f(0)=-f(1), \quad{ }_{q} D_{0^{+}}^{\alpha} f(0)=-{ }_{q} D_{0^{+}}^{\alpha} f(1),
\end{array}\right.
$$

for each $t \in[0,1]$, where $0<\alpha, \beta \leq 1, \eta$ is larger than or equal to zero, $1<\alpha+\beta<2, q \in$ $(0,1)$, and $\phi_{p}(s)=|s|^{p-2} s$, with $p \in(1,2]$. In 2017, Baleanu et al., presented a new method to investigate some fractional integro-differential equations involving the Caputo-Fabrizio derivation,

$$
{ }^{\mathrm{CF}} D^{\alpha} u(t)=\frac{(2-\alpha) M(\alpha)}{2(1-\alpha)} \int_{0}^{t} \exp \left(\frac{\alpha}{\alpha-1}(t-s)\right) u^{\prime}(s) d s
$$


where $t$ is used and $M(\alpha)$ is a normalization constant depending on $\alpha$ such that $M(0)=$ $M(1)=1$; one proved the existence of approximate solutions for these problems [16]. In the same year, they introduced a new operator entitled the infinite coefficient-symmetric Caputo-Fabrizio fractional derivative and applied it to the investigation of the approximate solutions for two infinite coefficient-symmetric Caputo-Fabrizio fractional integrodifferential problems [17].

In addition to, Akbari et al., by using the shifted Legendre and Chebyshev polynomials, discussed the existence of solutions for a sum-type fractional integro-differential problem under the Caputo differentiation [19]. Over the past three years, Baleanu and Rezapour et al., by using the Caputo-Fabrizio derivative, achieved innovation, and remarkable and interesting results were found for solutions of fractional differential equations [13-16, 18, 20-25]. In the next year, Rezapour et al., investigated the existence of solutions for the inclusion ${ }^{c} D^{\alpha} x(t) \in F\left(x, f(x),{ }^{c} D^{\beta} f(x), f^{\prime}(x)\right)$ for each $x \in I$ with the conditions ${ }^{c} D^{\beta} f(0)-$ $\int_{0}^{\eta_{1}} f(r) d r=f(0)+f^{\prime}(0)$ and ${ }^{c} D^{\beta} f(1)-\int_{0}^{\eta_{2}} f(r) d r=f(1)+f^{\prime}(1)$, where the multifunction $F$ maps $[0,1] \times \mathbb{R}^{3}$ to $2^{\mathbb{R}}$ and is compact valued and ${ }^{c} D^{\alpha}$ is the Caputo differential operator [54].

In 2019, Samei et al., discussed the fractional hybrid $q$-differential inclusions ${ }^{c} D_{q}^{\alpha}(x / F(t$, $\left.\left.x, I_{q}^{\alpha_{1}} x, \ldots, I_{q}^{\alpha_{n}} x\right)\right) \in T\left(t, x, I_{q}^{\beta_{1}} x, \ldots, I_{q}^{\beta_{k}} x\right)$, with the boundary conditions $x(0)=x_{0}$ and $x(1)=$ $x_{1}$, where $1<\alpha \leq 2, q \in(0,1), x_{0}, x_{1} \in \mathbb{R}, \alpha_{i}>0$, for $i=1,2, \ldots, n, \beta_{j}>0$, for $j=1,2, \ldots, k$, $n, k \in \mathbb{N},{ }^{c} D_{q}^{\alpha}$ denotes a Caputo type $q$-derivative of order $\alpha, I_{q}^{\beta}$ denotes the RiemannLiouville type $q$-integral of order $\beta, F: J \times \mathbb{R}^{n} \rightarrow(0, \infty)$ is continuous and $T$ mapping $J \times \mathbb{R}^{k}$ to $P(\mathbb{R})$ is a multifunction [32]. Also, they discussed the existence of solutions for the fractional $q$-derivative inclusions ${ }^{c} D_{q}^{\alpha} x(t) \in F\left(t, x(t), x^{\prime}(t),{ }^{c} D_{q}^{\beta} x(t)\right), x(0)+x^{\prime}(0)+{ }^{c} D_{q}^{\beta} x(0)=$ $\int_{0}^{\eta_{1}} x(s) d s$, and $x(1)+x^{\prime}(1)+{ }^{c} D_{q}^{\beta} x(1)=\int_{0}^{\eta_{2}} x(s) d s$, for any $t$ in $I$ and $q, \eta_{1}, \eta_{2}, \beta \in(0,1)$, where $F$ maps $I \times \mathbb{R}^{3}$ into $2^{\mathbb{R}}$ is a compact valued multifunction and ${ }^{c} D_{q}^{\alpha}$ is the fractional Caputo type $q$-derivative operator of order $\alpha \in(1,2]$, and $\Gamma_{q}(2-\beta)\left(\eta^{2} v-v^{2} \eta-\eta^{2}+v^{2}+4 \eta-\right.$ $2 v-2)+2(1-\eta) \neq 0$, such that $\alpha-\beta>1$ [49]. In 2019, Samei et al. [32, 36], investigated the fractional hybrid $q$-difference inclusion, and also equations and inclusions of multiterm fractional $q$-integro-differential equations with non-separated and initial boundary conditions.

In this article, motivated by the main idea of the literature, we are going to investigate the problems of the fractional $q$-differential equation

$$
\left\{\begin{array}{l}
\left({ }^{c} D_{q}^{\alpha} f\right)(t)=w\left(t, f(t), f^{\prime}(t),\left({ }^{c} D_{q}^{\beta} f\right)(t)\right) \\
f(0)=c_{1} f(1) \\
f^{\prime}(0)=c_{2}\left({ }^{c} D_{q}^{\beta} f\right)(1) \\
f^{\prime \prime}(0)=f^{\prime \prime \prime}(0)=\cdots=f^{(n-1)}(0)=0
\end{array}\right.
$$

where $\alpha \in(n-1, n)$ with $n \geq 3, \beta, q \in J=(0,1), c_{1} \in J, c_{2} \in(0, B)$ with $B=\Gamma_{q}(2-\beta)$, the function $w$ is $L^{\kappa}$-Carathéodory being positive real valued and $\kappa(\alpha-1)>1, w\left(t, x_{1}, x_{2}, x_{3}\right)$ may be singular at the value 0 of its space variables $x_{1}, x_{2}, x_{3} ;{ }^{c} D_{q}^{\alpha}$ is the fractional Caputo type $q$-derivative.

This manuscript is organized as follows: In Sect. 2, we recall some preliminary concepts and fundamental results of $q$-calculus. Section 3 is devoted to the main results, while examples illustrating the obtained results and algorithm for the problems are presented in Sect. 4. 


\section{Preliminaries}

First of all, we summarize the basic definitions and properties of $q$-calculus and $q$ fractional integrals and derivatives. One can find more information about them in $[1-6,8]$. Suppose that $q \in(0,1)$ and $a \in \mathbb{R}$. Define $[a]_{q}=\frac{1-q^{a}}{1-q}[1]$. The power function $(x-y)_{q}^{n}$ with $n \in \mathbb{N}_{0}$ is $(x-y)_{q}^{(n)}=\prod_{k=0}^{n-1}\left(x-y q^{k}\right)$ and $(x-y)_{q}^{(0)}=1$ where $x, y \in \mathbb{R}$ and $\mathbb{N}_{0}:=\{0\} \cup \mathbb{N}$ [1-3]. Also, for $\alpha \in \mathbb{R}$ and $a \neq 0$, we have $(x-y)_{q}^{(\alpha)}=x^{\alpha} \prod_{k=0}^{\infty}\left(x-y q^{k}\right) /\left(x-y q^{\alpha+k}\right)$. If $y=0$, then it is clear that $x^{(\alpha)}=x^{\alpha}$ (Algorithm 1). The $q$-Gamma function is given by $\Gamma_{q}(z)=(1-q)^{(z-1)} /(1-q)^{z-1}$, where $z \in \mathbb{R} \backslash\{0,-1,-2, \ldots\}[1,2,55,56]$. Note that $\Gamma_{q}(z+1)=$ $[z]_{q} \Gamma_{q}(z)$. We show in Algorithm 2, a pseudo-code for estimating the $q$-Gamma function. The $q$-derivative of the function $f$, is defined by $\left(D_{q} f\right)(x)=\frac{f(x)-f(q x)}{(1-q) x}$ and $\left(D_{q} f\right)(0)=$ $\lim _{x \rightarrow 0}\left(D_{q} f\right)(x)[2,6,57]$. One can find in Algorithm 3 a pseudo-code for calculating the $q$-derivative of the function $f$. The higher-order $q$-derivative of a function $f$ is defined by $\left(D_{q}^{n} f\right)(x)=D_{q}\left(D_{q}^{n-1} f\right)(x)$ for all $n \geq 1$, where $\left(D_{q}^{0} f\right)(x)=f(x)$ [57]. The $q$-integral of a function $f$ defined on $[0, b]$ is defined by

$$
I_{q} f(x)=\int_{0}^{x} f(s) d_{q} s=x(1-q) \sum_{k=0}^{\infty} q^{k} f\left(x q^{k}\right)
$$

for $x \in[0, b]$, provided that the series absolutely converges, which is shown in Algorithm 4 $[57,58]$. If $a$ is in $[0, b]$, then

$$
\int_{a}^{b} f(u) d_{q} u=I_{q} f(b)-I_{q} f(a)=(1-q) \sum_{k=0}^{\infty} q^{k}\left[b f\left(b q^{k}\right)-a f\left(a q^{k}\right)\right]
$$

whenever the series exists. The operator $I_{q}^{n}$ is given by $\left(I_{q}^{0} h\right)(x)=h(x)$ and

$$
\left(I_{q}^{n} h\right)(x)=\left(I_{q}\left(I_{q}^{n-1} h\right)\right)(x),
$$

for $n \geq 1$ and $g \in C([0, b])$ which is shown in Algorithm 5 [57]. It has been proved that $\left(D_{q}\left(I_{q} f\right)\right)(x)=f(x)$ and $\left(I_{q}\left(D_{q} f\right)\right)(x)=f(x)-f(0)$ whenever $f$ is continuous at $x=0[2,57$, 58]. The fractional Riemann-Liouville type $q$-integral of the function $f$ on $J$, of $\alpha \geq 0$ is given by $\left(I_{q}^{0} f\right)(t)=f(t)$ and

$$
\left(I_{q}^{\alpha} f\right)(t)=\frac{1}{\Gamma_{q}(\alpha)} \int_{0}^{t}(t-q s)^{(\alpha-1)} f(s) d_{q} s
$$

for $t \in J$ and $\alpha>0[35,55,59]$. Also, the fractional Caputo type $q$-derivative of the function $f$ is given by

$$
\begin{aligned}
\left({ }^{c} D_{q}^{\alpha} f\right)(t) & =\left(I_{q}^{[\alpha]-\alpha}\left(D_{q}^{[\alpha]} f\right)\right)(t) \\
& =\frac{1}{\Gamma_{q}([\alpha]-\alpha)} \int_{0}^{t}(t-q s)^{([\alpha]-\alpha-1)}\left(D_{q}^{[\alpha]} f\right)(s) d_{q} s,
\end{aligned}
$$

for $t \in J$ and $\alpha>0[35,59]$. It has been proved that $\left(I_{q}^{\beta}\left(I_{q}^{\alpha} f\right)\right)(x)=\left(I_{q}^{\alpha+\beta} f\right)(x)$ and $\left(D_{q}^{\alpha}\left(I_{q}^{\alpha} f\right)\right)(x)=f(x)$, where $\alpha$ and $\beta$ in $[0, \infty)[2,35,55,59]$.

Let $\bar{J}=[0,1]$ and $A$ be a subset of $\mathbb{R}^{3}$. We denote the space of functions whose $\kappa$ th powers of modulus are integrable on $\bar{J}$, endowed with norm $\|u\|_{\kappa}=\left(\int_{0}^{1}|u(t)|^{\kappa} d t\right)^{1 / \kappa}$ and the set of absolutely continuous functions on $\bar{J}$, by $L^{\kappa}(\bar{J})$ and $A C(\bar{J})$, respectively, where $\kappa \in[1, \infty)$. 
Definition 1 We say that $f$ is multi-singular when it is singular at more than one point $t$. Also, a real-valued and non-continuous function $f$ on the interval $I=[a, b]$ is said to be singular whenever $f$ is non-constant on $I$, and there exists a set $S$ of measure 0 such that for $x$ outside of $S$ the derivative $f^{\prime}(x)$ exists and is zero, that is, the derivative of $f$ vanishes almost everywhere.

Definition 2 A function $w$ is called $L^{\kappa}$-Carathéodory on $\bar{J} \times A$ whenever the real-valued function $w\left(\cdot, x_{1}, x_{2}, x_{3}\right)$ on $\bar{J}$ is measurable for all $\left(x_{1}, x_{2}, x_{3}\right)$ belonging to $A$, the real-valued function $w(t, \cdot, \cdot, \cdot)$ defined on $A$ is continuous for each $t$ and belongs to $(0,1]$ and for each compact set $C \subset A$, there exists $\varphi_{C} \in L^{\kappa}(\bar{J})$, such that $\left|w\left(t, x_{1}, x_{2}, x_{3}\right)\right| \leq \varphi_{C}(t)$, for $t$ belonging to $\bar{J}$ and $\left(x_{1}, x_{2}, x_{3}\right) \in C$.

Definition 3 A real value function $f$ define on $\bar{J}$ is called a positive solution for problem (1), whenever $f(t)$ is more than to zero, ${ }^{c} D_{q}^{\alpha} f$ is a function in $L^{k}(\bar{J})$ and $f$ satisfies the boundary conditions for all $t \in \bar{J}$.

Throughout the paper, we suppose that the function $w$ in (1) has the following conditions:

(H1) The map $w$ is an $L^{\kappa}$-Carathéodory on $\bar{J} \times A$, where $\kappa(\alpha-1)>1$ and it fulfills the estimate

$$
w\left(t, x_{1}, x_{2}, x_{3}\right) \leq g_{1}\left(x_{1}\right)+g_{2}\left(\left|x_{2}\right|\right)+g_{3}\left(\left|x_{3}\right|\right)+\gamma(t) \theta\left(x_{1},\left|x_{2}\right|,\left|x_{3}\right|\right),
$$

for $t \in \bar{J}$ and $\left(x_{1}, x_{2}, x_{3}\right)$ belonging to $A$, where positive valued functions $g_{1}, g_{2}, g_{3}$ in $C\left(\mathbb{R}^{>0}\right)$ are decreasing, $\gamma$ and $\theta$ in $L^{\kappa}(\bar{J})$ and $C(E)$ where

$E=[0, \infty) \times[0, \infty) \times[0, \infty)$, respectively, are positive, $w$ is increasing in all its

arguments and $\lim _{y \rightarrow \infty} \frac{w(y, y, y)}{y}=0$ and $\Gamma_{q}(\alpha)\left(I_{q}^{\alpha} g_{i}^{K}\right)(1)<\infty$ for $i=1,2,3$.

(H2) For each $t \in \bar{J}$ and $\left(x_{1}, x_{2}, x_{3}\right)$ belongs to $A$, there exists $m>0$ such that $m \leq w\left(t, x_{1}, x_{2}, x_{3}\right)$.

Since we imagine that problem (1) is singular, that is, $w\left(t, x_{1}, x_{2}, x_{3}\right)$ may be singular at the value zero of its space variables $x_{1}, x_{2}$ and $x_{3}$, we use regularization and sequential techniques for the existence of positive solutions of the problem. For this purpose, for each natural number $n$, define the function $w_{n}$ on $\bar{J} \times A$ by

$$
w_{n}\left(t, x_{1}, x_{2}, x_{3}\right)=w\left(t, \xi_{n}^{+}\left(x_{1}\right), \xi_{n}^{+}\left(x_{2}\right), \xi_{n}^{+}\left(x_{3}\right)\right),
$$

where $\xi_{n}^{+}(u)=u$, whenever $f \geq \frac{1}{n}$ and $\xi_{n}^{+}(u)=\frac{1}{n}$, whenever $u<\frac{1}{n}$.

Remark 1 Since $w$ is $L^{\kappa}$-Carathéodory, obviously $w_{n}$ is an $L^{\kappa}$-Carathéodory function on $\bar{J} \times A$ and by assumption (H1), for each $n$, we get

$$
\begin{aligned}
w_{n}\left(t, x_{1}, x_{2}, x_{3}\right) \leq & g_{1}\left(\frac{1}{n}\right)+g_{2}\left(\frac{1}{n}\right)+g_{3}\left(\frac{1}{n}\right) \\
& +\gamma(t) \theta\left(1+x_{1}, 1+\left|x_{2}\right|, 1+\left|x_{3}\right|\right)
\end{aligned}
$$

and $w_{n}\left(t, x_{1}, x_{2}, x_{3}\right) \leq g_{1}\left(x_{1}\right)+g_{2}\left(\left|x_{2}\right|\right)+g_{3}\left(\left|x_{3}\right|\right)+\gamma(t) \theta\left(1+x_{1}, 1+\left|x_{2}\right|, 1+\left|x_{3}\right|\right)$. Also, the condition (H2) entails that there exists a natural number $m$ such that $m \leq w_{n}\left(t, x_{1}, x_{2}, x_{3}\right)$. 
Lemma 4 ([60]) Suppose that $\tau$ belongs to $L^{\kappa}(\bar{J})$ and $t_{1}, t_{2} \in \bar{J}$. Then

$$
\left|\Gamma_{q}(\alpha-1)\left(I_{q}^{\alpha-1} \tau\right)(t)\right| \leq\left(\frac{t^{d}}{d}\right)^{1 / p}\|\tau\|_{\kappa}
$$

for almost all $t$ belongs to $\bar{J}$ and

$$
\begin{aligned}
& \left|\int_{0}^{t_{2}}\left(t_{2}-q s\right)^{(\alpha-2)} \tau(s) d_{q} s-\int_{0}^{t_{1}}\left(t_{1}-q s\right)^{(\alpha-2)} \tau(s) d_{q} s\right| \\
& \quad \leq\left(\frac{t_{1}^{d}+\left(t_{2}-t_{1}\right)^{d}-t_{2}^{d}}{d}\right)^{1 / p}\|\tau\|_{\kappa}+\left(\frac{\left(t_{2}-t_{1}\right)^{d}}{d}\right)^{1 / p}\|\tau\|_{\kappa},
\end{aligned}
$$

whenever $t_{1} \leq t_{2}$, here $d-1=(\alpha-2) p$ with $p=\frac{\kappa-1}{\kappa}$.

\section{Main results}

At present, we discuss the existence of solutions of problem (1). Foremost, we prove the key result.

Lemma 5 Suppose that $v$ belongs to $C(\bar{J})$. Then the boundary value problem

$$
\left\{\begin{array}{l}
\left({ }^{c} D_{q}^{\alpha} f\right)(t)=v(t), \\
f(0)=c_{1} f(1), \\
f^{\prime}(0)=c_{2}\left({ }^{c} D_{q}^{\beta} f\right)(1), \\
f^{\prime \prime}(0)=f^{\prime \prime \prime}(0)=\cdots=f^{(n-1)}(0)=0,
\end{array}\right.
$$

for each $t \in J$, where $c_{1} \in(n-1, n)$ with $n \geq 3$ and $c_{2} \in(0, B)$ with $B=\Gamma_{q}(2-\beta)$, is equivalent to the fractional integral equation $f(t)=\int_{0}^{1} G_{q}(t, s) v(s) d_{q} s$, for all $s, t \in \bar{J}$, where

$$
G_{q}(t, s)= \begin{cases}\frac{(t-q s)^{(\alpha-1)}}{\Gamma_{q}(\alpha)}+\frac{c_{1}(1-q s)^{(\alpha-1)}}{\left(1-c_{1}\right) \Gamma_{q}(\alpha)}+\frac{c_{2} B\left(c_{1}+t-c_{1} t\right)(1-q s)(\alpha-\beta-1)}{(1-a) \Gamma_{q}(\alpha-\beta)\left(B-c_{2}\right)}, & s \leq t, \\ \frac{c_{1}(1-q s)^{(\alpha-1)}}{\left(1-c_{1}\right) \Gamma_{q}(\alpha)}+\frac{c_{2} B\left(c_{1}+t-c_{1} t\right)(1-q s)(\alpha-\beta-1)}{\left(1-c_{1}\right) \Gamma_{q}(\alpha-\beta)\left(B-c_{2}\right)}, & t \leq s .\end{cases}
$$

Proof From $\left({ }^{c} D_{q}^{\alpha}\right) f(t)=v(t)$, for all $t$ belonging to $(0,1)$ and the boundary conditions $f^{\prime \prime}(0)=f^{\prime \prime \prime}(0)=f^{(n-1)}(0)=0$, we obtain

$$
\begin{aligned}
f(t) & =\left(I_{q}^{\alpha} v\right)(t)+f(0)+f^{\prime}(0) t+\frac{f^{\prime \prime}(0)}{2 !} t^{2}+\cdots+\frac{f^{(n-1)}(0)}{(n-1) !} t^{n-1} \\
& =\left(I_{q}^{\alpha} v\right)(t)+f(0)+f^{\prime}(0) t .
\end{aligned}
$$

So, we obtain

$$
\left({ }^{c} D_{q}^{\beta} f\right)(t)=\left(I_{q}^{\alpha-\beta} v\right)(t)+\left({ }^{c} D_{q}^{\beta}\right)\left(f(0)+f^{\prime}(0) t\right)=\left(I_{q}^{\alpha-\beta} v\right)(t)+\frac{1}{B} f^{\prime}(0) t^{1-\beta} .
$$

Therefore, $f(1)=\left(I_{q}^{\alpha} v\right)(1)+f(0)+f^{\prime}(0)$, and $\left({ }^{c} D_{q}^{\beta} f\right)(1)=\left(I_{q}^{\alpha-\beta} v\right)(1)+\frac{1}{B} f^{\prime}(0)$. By using the conditions of problem (3), we get $f(0)=c_{1}\left(\left(I_{q}^{\alpha} v\right)(1)+f(0)+f^{\prime}(0)\right)$ and $f^{\prime}(0)=c_{2}\left(\left(I_{q}^{\alpha-\beta} v\right)(1)+\right.$ 
$\left.\frac{1}{B} f^{\prime}(0)\right)$. Hence,

$$
f(0)=\frac{c_{1}}{\left(1-c_{1}\right)}\left(I_{q}^{\alpha} v\right)(1)+\frac{c_{1} c_{2} B}{\left(1-c_{1}\right)\left(B-c_{2}\right)}\left(I_{q}^{\alpha-\beta} v\right)(1)
$$

and $f^{\prime}(0)=\frac{c_{2} B}{B-c_{2}}\left(I_{q}^{\alpha-\beta} v\right)(1)$. We simply observe that

$$
\begin{aligned}
f(t)= & \left(I_{q}^{\alpha} v\right)(t) d_{q} s+f(0)+f^{\prime}(0) t \\
= & \int_{0}^{t}\left[\frac{(t-q s)^{(\alpha-1)}}{\Gamma_{q}(\alpha)}+\frac{c_{1}(1-q s)^{(\alpha-1)}}{\left(1-c_{1}\right) \Gamma_{q}(\alpha)}\right. \\
& \left.+\frac{c_{2} B\left(c_{1}+t-c_{1} t\right)(1-q s)^{(\alpha-\beta-1)}}{\left(1-c_{1}\right) \Gamma_{q}(\alpha-\beta)\left(B-c_{2}\right)}\right] v(s) d_{q} s \\
& +\int_{t}^{1}\left[\frac{c_{1}(1-q s)^{(\alpha-1)}}{\left(1-c_{1}\right) \Gamma_{q}(\alpha)}\right. \\
& \left.+\frac{c_{2} B\left(c_{1}+t-c_{1} t\right)(1-q s)^{(\alpha-\beta-1)}}{\left(1-c_{1}\right) \Gamma_{q}(\alpha-\beta)\left(B-c_{2}\right)}\right] v(s) d_{q} s \\
= & \int_{0}^{1} G_{q}(t, s) v(s) d_{q} s .
\end{aligned}
$$

This completes our proof.

For unification, we put $p=\frac{\kappa-1}{\kappa}$ with $\kappa \geq 1, d=(\alpha-2) p+1$,

$$
\begin{aligned}
\Lambda_{1} & =\frac{1}{\left(1-c_{1}\right) \Gamma_{q}(\alpha)}+\frac{\Gamma_{q}(\alpha-\beta)\left(B-c_{2}\right)+c_{2} \Gamma_{q}(2-\beta)}{\left(1-c_{1}\right) \Gamma_{q}(\alpha-\beta)\left(\Gamma_{q}(2-\beta)-c_{2}\right)} \\
& =\frac{\Gamma_{q}(\alpha-\beta)\left(B-c_{2}\right)+c_{2} B \Gamma_{q}(\alpha)}{\left(1-c_{1}\right) \Gamma_{q}(\alpha) \Gamma_{q}(\alpha-\beta)\left(B-c_{2}\right)}
\end{aligned}
$$

and

$$
\Lambda_{2}=\frac{c_{1} c_{2} B}{\left(1-c_{1}\right) \Gamma_{q}(\alpha-\beta)\left(B-c_{2}\right)} .
$$

Lemma 6 The $q$-Green function $G_{q}(t, s)$ in Lemma 5, which belongs to $C(\bar{J} \times \bar{J})$, for all $(t, s) \in \bar{J} \times \bar{J}$, satisfies the conditions:

(i) $G_{q}(t, s) \leq \Lambda_{1}(1-q s)^{(\alpha-\beta-1)} \leq 1$,

(ii) $G_{q}(t, s) \geq \Lambda_{2}(1-q s)^{(\alpha-\beta-1)}$.

Proof One can easy to check that $G_{q}(t, s)>0$ on $\bar{J} \times \bar{J}$. Then from (5) and (6), we have

$$
\begin{aligned}
& \frac{(t-q s)^{(\alpha-1)}}{\Gamma_{q}(\alpha)}+\frac{c_{1}(1-q s)^{(\alpha-1)}}{\left(1-c_{1}\right) \Gamma_{q}(\alpha)}+\frac{c_{2} B\left(c_{1}+t-c_{1} t\right)(1-q s)^{(\alpha-\beta-1)}}{\left(1-c_{1}\right) \Gamma_{q}(\alpha-\beta)\left(B-c_{2}\right)} \\
& \quad \leq \frac{(1-q s)^{(\alpha-\beta-1)}\left(\Gamma_{q}(\alpha-\beta)(B)-c_{2}\right)+c_{2} B \Gamma_{q}(\alpha)}{\left(1-c_{1}\right) \Gamma_{q}(\alpha) \Gamma_{q}(\alpha-\beta)\left(B-c_{2}\right)} \\
& =\Lambda_{1}(1-q s)^{(\alpha-\beta-1)}
\end{aligned}
$$


and

$$
\begin{aligned}
& \frac{c_{1}(1-q s)^{(\alpha-1)}}{\left(1-c_{1}\right) \Gamma_{q}(\alpha)}+\frac{c_{2} B\left(c_{1}+t-c_{1} t\right)(1-q s)^{(\alpha-\beta-1)}}{\left(1-c_{1}\right) \Gamma_{q}(\alpha-\beta)\left(B-c_{2}\right)} \\
& \leq \frac{(1-q s)^{(\alpha-\beta-1)}\left(c_{1}(1-q s)^{(-\beta)}\right.}{\left(1-c_{1}\right) \Gamma_{q}(\alpha)} \\
& \quad+\frac{c_{2} B\left(c_{1}+t-c_{1} t\right)}{\left(1-c_{1}\right) \Gamma_{q}(\alpha-\beta)\left(B-c_{2}\right)} \\
& \leq \Lambda_{1}(1-q s)^{(\alpha-\beta-1)},
\end{aligned}
$$

whenever $s \leq t$ and $t \leq s$, respectively, for $t$ and $s$ in $\bar{J}$. Therefore, $G_{q}(t, s) \leq \Lambda_{1}(1-$ $q s)^{(\alpha-\beta-1)}$, for all $(t, s)$ belonging to $\bar{J} \times \bar{J}$. Finally, it is observed that

$$
\begin{aligned}
& \left(1-c_{1}\right)\left(B-c_{2}\right) \Gamma_{q}(\alpha-\beta) \Gamma_{q}(\alpha) G_{q}(t, s) \\
& \quad \geq c_{2} B \Gamma_{q}(\alpha)\left(c_{1}+t-c_{1} t\right)(1-q s)^{(\alpha-\beta-1)} \\
& \quad \geq c_{1} c_{2} B \Gamma_{q}(\alpha)(1-q s)^{(\alpha-\beta-1)} .
\end{aligned}
$$

Therefore, $G_{q}(t, s) \geq \Lambda_{2}(1-q s)^{(\alpha-\beta-1)}$ for all $(t, s)$ belonging to $\bar{J} \times \bar{J}$.

Consider the Banach space $X=C^{1}(\bar{J})$ endowed with the norm $\|u\|_{*}=\max \left\{\|u\|,\left\|u^{\prime}\right\|\right\}$ and the cone $P$ on $X$, containing all the functions $u$ belonging to $X$ such that $u(t) \geq 0$ and $u^{\prime}(t) \geq 0$ for all $t$. Now, we define an operator $\Theta_{n}$ on $P$ by

$$
\left(\Theta_{n} u\right)(t)=\int_{0}^{1} G_{q}(t, s) T_{n}\left(s, f(s), f^{\prime}(s),\left({ }^{c} D_{q}^{\beta} f\right)(s)\right) d_{q} s .
$$

At present, we show that the operator $\Theta_{n}$ is completely continuous [61].

Lemma $7 \Theta_{n}$ is a completely continuous operator, whenever the $\Theta_{n}$ satisfy conditions $(H 1)$ and (H2) for all natural number sn.

Proof Consider an element $u \in P$. Then $u \in C(\bar{J})$. Also, $u$ and $u^{\prime}$ are larger than or equal to zero. Therefore by the definition of ${ }^{c} D_{q}^{\beta}$, we get $\left({ }^{c} D_{q}^{\beta} u\right)(t) \in C(\bar{J})$ and $\left({ }^{c} D_{q}^{\beta} u\right)(t) \geq 0$. Now, define $\tau(t)=w_{n}\left(t, f(t), f^{\prime}(t),\left({ }^{c} D_{q}^{\beta} f\right)(t)\right)$. Then $\tau \in L^{\kappa}(\bar{J})$ and $\tau(t)$ higher than or equal to $m$ for almost all $t \in \bar{J}$. It follows from $G_{q}(t, s) \geq 0$ for all $(t, s)$ belonging to $\bar{J} \times \bar{J}$, from the equality

$$
\begin{aligned}
\left(\Theta_{n} u\right)(t)= & \frac{a \Gamma_{q}(\alpha-\beta)\left(B-c_{2}\right)(1-q s)^{(\alpha-1)}}{\left(1-c_{1}\right) \Gamma_{q}(\alpha-\beta)\left(\Gamma_{q}(2-\beta)-c_{2}\right)}\left(I_{q}^{\alpha} \tau\right)(1) \\
& +\frac{c_{2} B \Gamma_{q}(\alpha)\left(c_{1}+t-c_{1} t\right)(1-q s)^{(\alpha-\beta-1)}}{\left(1-c_{1}\right) \Gamma_{q}(\alpha)\left(B-c_{2}\right)}\left(I_{q}^{\alpha-\beta} \tau\right)(1)+\left(I_{q}^{\alpha} \tau\right)(t) .
\end{aligned}
$$

From the properties of $I_{q}^{\alpha}$ that $\Theta_{n} u \in C(\bar{J})$ and $\left(\Theta_{n} u\right)(t) \geq 0$ for all $t \in \bar{J}$ we have $\left(\Theta_{n} u\right)^{\prime}(t)=$ $\left(I_{q}^{\alpha-1} \tau\right)(t)$. Hence, $\left(\Theta_{n} u\right)^{\prime} \in C(\bar{J})$ and $\left(\Theta_{n} u\right)^{\prime}$ higher than or equal to zero, on $\bar{J}$. We test that the operator $\Theta_{n}$ is continuous. Suppose that the sequence $u_{m} \subset P$ is convergent and 
$\lim _{m \rightarrow \infty} u_{m}=u$. Thus, $\lim _{m \rightarrow \infty} u_{m}^{(i)}(t)=u^{(i)}(t)$ uniformly on $\bar{J}$ for $i=0$, 1 . Since

$$
\left({ }^{c} D_{q}^{\beta} u\right)(t)=\frac{d}{d t}\left(I_{q}^{1-\beta}\right)(u(t)-u(0))=\left(I_{q}^{1-\beta} u^{\prime}\right)(t)
$$

we get

$$
\left|\left({ }^{c} D_{q}^{\beta} u_{m}\right)(t)-\left({ }^{c} D_{q}^{\beta} u\right)(t)\right| \leq \frac{\left\|u_{m}^{\prime}-u^{\prime}\right\|}{\Gamma_{q}(1-\beta)} \int_{0}^{t}(t-q s)^{(-\beta)} d_{q} s \leq \frac{\left\|u_{m}-u\right\|_{*}}{\Gamma_{q}(\beta)}
$$

and $\lim _{m \rightarrow \infty}\left({ }^{c} D_{q}^{\beta} u_{m}\right)(t)=\left({ }^{c} D_{q}^{\beta} u\right)(t)$ uniformly on $\bar{J}$. In addition, by using (7), we have $\left|\left({ }^{c} D_{q}^{\beta} u_{m}\right)(t)\right| \leq \frac{u_{m}^{\prime}}{\Gamma_{q}(\beta)}$ and so

$$
\left\|\left({ }^{c} D_{q}^{\beta} u_{m}\right)\right\| \leq \frac{\left\|u_{m}^{\prime}\right\|}{\Gamma_{q}(\beta)} .
$$

Put $\tau_{m}(t)=w_{n}\left(t, u_{m}(t), u_{m}^{\prime}(t),\left({ }^{c} D_{q}^{\beta} u_{m}\right)(t)\right)$ and $\tau(t)=w_{n}\left(t, u(t), u^{\prime}(t),\left({ }^{c} D_{q}^{\beta} u\right)(t)\right)$. Then $\lim _{m \rightarrow \infty} \tau_{m}(t)=\tau(t)$ and there exists $\mu \in L^{\kappa}(\bar{J})$ such that $0 \leq \tau_{m}(t) \leq \mu(t)$, for each $t$ in $\bar{J}$ and natural number $m$. Since $w_{n}$ is a $L^{\kappa}$-Carathéodory function, $\left\{u_{m}\right\},\left\{\left({ }^{c} D_{q}^{\beta} u_{m}\right)(t)\right\}$ are bounded in $C^{1}(\bar{J}), C(\bar{J})$, respectively. So, $\lim _{m \rightarrow \infty}\left(\Theta_{n} u_{m}\right)(t)=\left(\Theta_{n} u\right)(t)$ uniformly on $\bar{J}$. Since $\left\{\tau_{m}\right\}$ is $L^{\kappa}$-convergent on $\bar{J}$, we conclude that $\lim _{m \rightarrow \infty}\left(\Theta_{n} u_{m}\right)^{\prime}(t)=$ $\lim _{m \rightarrow \infty}\left(I_{q}^{\alpha-1} \tau_{m}\right)(t)=\left(\Theta_{n} u\right)^{\prime}(t)$, uniformly on $\bar{J}$. Hence, the operator $\Theta_{n}$ is a continuous. We choose a positive constant $r$ such that both $\left\|u_{m}\right\|$ and $\left\|u_{m}^{\prime}\right\|$ are less than or equal to $r$ for each natural number $m$, thus, we have $\Gamma_{q}(\beta)\left\|\left({ }^{c} D_{q}^{\beta} u_{m}\right)(t)\right\| \leq r$ and

$$
\begin{aligned}
\left|\int_{0}^{t}(t-q s)^{(\alpha-2)} \tau_{m}(s) d_{q} s\right| & \leq\left(\int_{0}^{t}(t-q s)^{((\alpha-2) p)} d_{q} s\right)^{\frac{1}{p}}\left(\int_{0}^{t}\left|\tau_{m}(s)\right|^{\kappa} d_{q} s\right)^{\frac{1}{\kappa}} \\
& \leq\left(\frac{t^{d}}{d}\right)^{\frac{1}{p}}\left\|\tau_{m}\right\|_{\kappa},
\end{aligned}
$$

for all $m$. On the other hand, the relations

$$
0 \leq\left(\Theta_{n} u_{m}\right)(t)=\int_{0}^{1} G_{q}(t, s) \tau_{m}(s) d_{q} s \leq \int_{0}^{1} G_{q}(t, s) \mu(s) d_{q} s \leq \frac{\|\mu\|_{1}}{\Gamma_{q}(\alpha)}
$$

and

$$
0 \leq\left(\Theta_{n} u_{m}\right)^{\prime}(t)=\left(I_{q}^{\alpha-1} \tau_{m}\right)(t) \leq\left(I_{q}^{\alpha-1} \mu\right)(t) \leq \frac{1}{\Gamma_{q}(\alpha-1)}\left[\frac{1}{(\alpha-2) p+1}\right]^{\frac{1}{p}}\|\mu\|_{\kappa},
$$

hold for each $t$ and $m$ and so $\left\{\Theta_{n} u_{m}\right\}$ is bounded in $C(\bar{J})$. Moreover, it follows from Lemma 4 that

$$
\begin{aligned}
\left|\left(\Theta_{n} u_{m}\right)^{\prime}\left(t_{2}\right)-\left(\Theta_{n} u_{m}\right)^{\prime}\left(t_{1}\right)\right| & =\left|\left(I_{q}^{\alpha-1}\right)\left(\tau_{m}\left(t_{2}\right)-\tau_{m}\left(t_{1}\right)\right)\right| \\
& \leq \frac{\left\|\tau_{m}\right\|_{\kappa}}{\Gamma_{q}(\alpha-1)}\left[\left(\frac{t_{1}^{d}+\left(t_{2}-t_{1}\right)^{d}-t_{2}^{d}}{d}\right)^{\frac{1}{p}}+\left(\frac{\left(t_{2}-t_{1}\right)^{d}}{d}\right)^{\frac{1}{p}}\right] \\
& \leq \frac{\|\mu\|_{\kappa}}{\Gamma_{q}(\alpha-1)}\left[\left(\frac{t_{1}^{d}+\left(t_{2}-t_{1}\right)^{d}-t_{2}^{d}}{d}\right)^{\frac{1}{p}}+\left(\frac{\left(t_{2}-t_{1}\right)^{d}}{d}\right)^{\frac{1}{p}}\right],
\end{aligned}
$$


for each $t_{1}$ and $t_{2}$ belonging to $\bar{J}$ such that $t_{1} \leq t_{2}$ is fulfilled. As a result, $\left\{\left(\Theta_{n} u_{m}\right)^{\prime}\right\}$ is equicontinuous on $\bar{J}$. Consequently, based on the Arzelà-Ascoli theorem, $\left\{\Theta_{n} u_{m}\right\}$ is relatively compact in $C^{1}(\bar{J})$. Also, since $\Theta_{n}$ is continuous, we conclude that the operator $\Theta_{n}$ is completely continuous.

Lemma $8([61,62])$ Let $X$ be a Banach space, $P \subset X$ a cone and $\mathcal{O}_{1}$ and $\mathcal{O}_{2}$ bounded open balls in $X$ centered at the origin with $\overline{\mathcal{O}}_{1} \subset \mathcal{O}_{2}$. A completely continuous operator $w$ mapping $P \cap\left(\overline{\mathcal{O}}_{2} \backslash \mathcal{O}_{1}\right)$ into $P$ has a fixed point whenever $\|w(u)\| \geq\|u\|$ and $\|w(u)\| \leq\|u\|$ for $u \in P \cap \partial \mathcal{O}_{1}$ and $u \in P \cap \partial \mathcal{O}_{2}$, respectively.

Theorem 9 Let $w$ satisfy conditions (H1) and (H2). Then problem (1) has a solution $f_{n}$ in P such that

$$
f_{n} \geq \frac{m \Lambda_{2}}{\alpha-\beta}, \quad f_{n}^{\prime}(t) \geq \frac{m t^{\alpha-1}}{\Gamma_{q}(\alpha)}, \quad \text { and } \quad\left({ }^{c} D_{q}^{\beta} f_{n}\right)(t) \geq \frac{m t^{\alpha-\beta}}{\Gamma_{q}(\alpha-\beta+1)},
$$

for all t belonging to $\bar{J}$ and the natural number $n$.

Proof By using Lemma 7, one can conclude that the operator $\Theta_{n}: P \rightarrow P$ is completely continuous. A function $f$ is a solution of problem (1), whenever $f$ solves the operator equation $f=\Theta_{n} f$. Finally, we demonstrate $w_{n}$ in $P$ is a fixed point of $\Theta_{n}$ with desired continuousness. For this purpose, it is observed that

$$
\begin{aligned}
\left(\Theta_{n} u\right)(t) & =\int_{0}^{1} G_{q}(t, s) w_{n}\left(s, u(s), u^{\prime}(s),\left({ }^{c} D_{q}^{\beta} u\right)(s)\right) d_{q} s \\
& \geq m \int_{0}^{1} G_{q}(t, s) d_{q} s \geq m \int_{0}^{1}(1-t)^{\alpha}(1-q s)^{(\alpha-\beta-1)} d_{q} s \\
& =\frac{m \Lambda_{2}}{\alpha-\beta}
\end{aligned}
$$

and so $\left\|\Theta_{n} u\right\|_{*} \geq\left\|\Theta_{n} u\right\| \geq \frac{m \Lambda_{2}}{\alpha-\beta}$. Put

$$
\mathcal{O}_{1}=\left\{u \in X:\|u\|_{*}<\frac{m \Lambda_{2}}{\alpha-\beta}\right\}
$$

Then $\left\|\Theta_{n} u\right\|_{*} \geq\|u\|_{*}$ for all $u$ belonging to $P \cap \partial \mathcal{O}_{1}$. Let $v_{n}=g_{1}\left(\frac{1}{n}\right)+g_{2}\left(\frac{1}{n}\right)+g_{3}\left(\frac{1}{n}\right)$. Inequality (7) implies that

$$
\begin{aligned}
\left|\left(\Theta_{n} u\right)(t)\right| & \leq\left|\int_{0}^{1} G_{q}(t, s) w_{n}\left(s, f(s), f^{\prime}(s),\left({ }^{c} D_{q}^{\beta} f\right)(s)\right) d_{q} s\right| \\
& \leq \int_{0}^{1}\left|G_{q}(t, s)\right|\left[v_{n}+\gamma(s) \theta\left(1+|u(s)|, 1+\left|u^{\prime}(s)\right|, 1+\left|\left({ }^{c} D_{q}^{\beta} u\right)(s)\right|\right)\right] d_{q} s \\
& \leq \Lambda_{1}\left(v_{n}+w\left(1+\|u\|, 1+\left\|u^{\prime}\right\|, 1+\left\|\left({ }^{c} D^{\beta} u\right)\right\|\right)\|\gamma\|_{1}\right)
\end{aligned}
$$

and

$$
\begin{aligned}
\left|\left(\Theta_{n} u\right)^{\prime}(t)\right| & =\left|\left(I_{q}^{\alpha-1} w_{n}\right)\left(t, u(t), u^{\prime}(t),\left({ }^{c} D_{q}^{\beta} u\right)(t)\right) d_{q} s\right| \\
& \leq\left(I_{q}^{\alpha-1}\left(v_{n}+\gamma(t) \theta\left(1+|u(t)|, 1+\left|u^{\prime}(t)\right|, 1+\left|\left({ }^{c} D_{q}^{\beta} u\right)(t)\right|\right)\right)\right)
\end{aligned}
$$




$$
\begin{aligned}
& +\frac{v_{n} t^{\alpha-1}}{(\alpha-1) \Gamma_{q}(\alpha-1)} \\
& +w\left(1+\|u\|, 1+\left\|u^{\prime}\right\|, 1+\left\|\left({ }^{c} D_{q}^{\beta} u\right)\right\|\right)\left(I_{q}^{\alpha-1} \gamma\right)(t),
\end{aligned}
$$

for each $u \in P$ and all $t \in \bar{J}$, because $w$ is increasing in all its arguments. Since $\|u\|$ and $\left\|u^{\prime}\right\|$ are less than or equal to $\|u\|_{*},\left\|\left({ }^{c} D_{q}^{\beta} u\right)\right\| \leq \frac{\left\|u^{\prime}\right\|}{\Gamma_{q}(\beta)} \leq \frac{\|u\|_{*}}{\Gamma_{q}(\beta)}$ and by inequality (9), $\int_{0}^{t}(t-$ $q s)^{(\alpha-2)} \gamma(s) d_{q} s \leq\left(\frac{1}{d}\right)^{1 / p}\|\gamma\|_{\kappa}$, we have

$$
\left\|\Theta_{n}(x)\right\| \leq \Lambda_{1}\left[v_{n}+w\left(1+\|u\|_{*}, 1+\|u\|_{*}, 1+\frac{\|u\|_{*}}{\Gamma_{q}(\beta)}\right)\|\gamma\|_{1}\right]
$$

and

$$
\left\|\left(\Theta_{n} u\right)^{\prime}\right\| \leq \frac{1}{\Gamma_{q}(\alpha-1)}\left[\frac{v_{n}}{\alpha-1}+w\left(1+\|u\|_{*}, 1+\|u\|_{*}, 1+\frac{\|u\|_{*}}{\Gamma_{q}(\beta)}\right)\left(\frac{1}{d}\right)^{1 / p}\|\gamma\|_{\kappa}\right]
$$

Therefore,

$$
\left\|\Theta_{n} u\right\|_{*} \leq M\left[\frac{v_{n}}{\alpha-1}+N w\left(1+\|u\|_{*}, 1+\|u\|_{*}, 1+\frac{\|u\|_{*}}{\Gamma_{q}(\beta)}\right)\right]
$$

where $N$ and $M$ are $\max \left\{\|\gamma\|_{1},\left(\frac{1}{d}\right)^{1 / p}\|\gamma\|_{\kappa}\right\}$ and $\max \left\{\Lambda_{1}, \frac{1}{\Gamma_{q}(\alpha-1)}\right\}$, respectively. Since

$$
\lim _{v \rightarrow \infty} \frac{w(1+v, 1+v, 1+v)}{v}
$$

is equal to zero, by condition (H1), there exists a positive constant $L$ such that

$$
M\left[\frac{v_{n}}{\alpha-1}+N w\left(1+v, 1+v, \frac{v}{\Gamma(\beta)}\right)\right]<v,
$$

for each $v$ higher than or equal to $L$. Hence, $\left\|\Theta_{n} u\right\|_{*}<\|u\|_{*}$ for all $u$ in $P$ with $\|u\|_{*} \geq L$. Let $\mathcal{O}_{2}=\left\{u \in X:\|u\|_{*}<L\right\}$, then $\left\|\theta_{n} u\right\|_{*}<\|u\|_{*}$ for $u \in P \cap \partial \Omega_{2}$. Now applying the last result, with $X$ and $w=\Theta_{n}$, we conclude that $\Theta_{n}$ has a fixed point $f_{n}$ in $P \cap\left(\overline{\mathcal{O}}_{2} \backslash \mathcal{O}_{1}\right)$. Consequently, $f_{n}$ is a solution of Problem (1). The first inequality follows from (11), $f_{n}=\left(\Theta_{n} f_{n}\right)(t) \geq \frac{m \Lambda_{2}}{\alpha-\beta}$, the second one follows from the relation

$$
\left(\Theta_{n} u\right)^{\prime}(t)=\left(I_{q}^{\alpha-1} w_{n}\right)\left(t, u(t), u^{\prime}(t),\left({ }^{c} D_{q}^{\beta} u\right)(t)\right) \geq\left(I_{q}^{\alpha-1} m\right)=\frac{m t^{\alpha-1}}{\Gamma_{q}(\alpha)}
$$

for $t \in \bar{J}$ and $u$ belongs to $P$. Finally, using the second inequality and $\left(I_{q}^{1-\beta} u\right)(t)=$ $\frac{\Gamma_{q}(\alpha)}{\Gamma_{q}(\alpha-\beta+1)} t^{\alpha-\beta}$, where $u(t)=t^{\alpha-1}$, we obtain

$$
\left({ }^{c} D_{q}^{\beta} f_{n}\right)(t)=\left(I_{q}^{1-\beta} f_{n}^{\prime}\right)(t) \geq \frac{m}{\Gamma_{q}(\alpha)}\left(I_{q}^{1-\beta} h\right)(t)=\frac{m t^{\alpha-\beta}}{\Gamma_{q}(\alpha-\beta+1)},
$$

for each $t$. This completes our proof.

Theorem 10 The problem (1) has a solution $f$ such that $(\alpha-\beta) f(t) \geq m \Lambda_{2}, \Gamma_{q}(\alpha) f^{\prime}(t) \geq$ $m t^{\alpha-1}$ and $\Gamma_{q}(\alpha-\beta+1)\left({ }^{c} D_{q}^{\beta} f\right)(t) \geq m t^{\alpha-\beta}$, for all $t \in \bar{J}$, whenever conditions $(\mathrm{H} 1)$ and $(\mathrm{H} 2)$ hold. 
Liang and Same Advances in Difference Equations

(2020) 2020:14

Page 12 of 22

Proof By using Theorem 9, for each $n$, problem (1) has a solution $f_{n} \in P$ which satisfies inequality (10). Hence

$$
g_{1}\left(f_{n}(t)\right) \leq g_{1}\left(\frac{m \Lambda_{2}}{\alpha-\beta}\right), \quad g_{2}\left(\left|f_{n}^{\prime}(t)\right|\right) \leq g_{2}\left(\frac{m t^{\alpha-1}}{\Gamma_{q}(\alpha)}\right)
$$

and

$$
g_{3}\left(\left|\left({ }^{c} D_{q}^{\beta} f_{n}\right)(t)\right|\right) \leq g_{3}\left(\frac{m t^{\alpha-\beta}}{\Gamma_{q}(\alpha-\beta+1)}\right)
$$

for each $t \in \bar{J}$ and all natural number $n$. In addition, it follows from (8) that $\left\|\left({ }^{c} D_{q}^{\beta} f_{n}\right)\right\| \leq$ $\frac{\left\|f_{n}^{\prime}\right\|}{\Gamma_{q}(\beta)}$. We put

$$
F(t)=g_{1}\left(\frac{m \Lambda_{2}}{\alpha-\beta}\right)+g_{2}\left(\frac{m t^{\alpha-1}}{\Gamma_{q}(\alpha)}\right)+g_{3}\left(\frac{m t^{\alpha-\beta}}{\Gamma_{q}(\alpha-\beta+1)}\right) .
$$

Therefore, we conclude that

$$
\begin{aligned}
m & \leq w_{n}\left(t, f_{n}(t), f_{n}^{\prime}(t),\left({ }^{c} D_{q}^{\beta} f_{n}\right)(t)\right) \\
& \leq F(t)+\gamma(t) \theta\left(1+\left\|f_{n}\right\|, 1+\left\|f_{n}^{\prime}\right\|, 1+\left\|\left({ }^{c} D_{q}^{\beta} f_{n}\right)\right\|\right) \\
& \leq F(t)+\gamma(t) \theta\left(1+\left\|f_{n}\right\|_{*}, 1+\left\|f_{n}^{\prime}\right\|_{*}, 1+\frac{\left\|f_{n}\right\|_{*}}{\Gamma_{q}(\beta)}\right) .
\end{aligned}
$$

Since we have a positive value $G_{q}(t, s) \leq \Lambda_{1}$, we get

$$
\begin{aligned}
0 & \leq f_{n}(t)=\int_{0}^{1} G_{q}(t, s) w_{n}\left(s, f_{n}(s), f_{n}^{\prime}(s),\left({ }^{c} D_{q}^{\beta} f_{n}\right)(s)\right) d_{q} s \\
& \leq \Lambda_{1}\left[\int_{0}^{1} F(q s) d_{q} s+w\left(1+\left\|f_{n}\right\|_{*}, 1+\left\|f_{n}\right\|_{*}, 1+\frac{\left\|f_{n}\right\|_{*}}{\Gamma_{q}(\beta)}\right)\|\gamma\|_{1}\right]
\end{aligned}
$$

and

$$
0 \leq f_{n}^{\prime}(t) \leq\left(I_{q}^{\alpha-1} F\right)(t)+w\left(1+\left\|f_{n}\right\|_{*}, 1+\left\|f_{n}\right\|_{*}, 1+\frac{\left\|f_{n}\right\|_{*}}{\Gamma_{q}(\beta)}\right)\left(I_{q}^{\alpha-1} \gamma\right)(t)
$$

At present, we show that $\int_{0}^{t}(t-q s)^{(\alpha-2)} F(s) d_{q} s$ is bounded on $[0,1]$. By using the Hölder inequality, we get

$$
\begin{aligned}
& \int_{0}^{1}(t-q s)^{(\alpha-2)} g_{1}\left(\frac{m \Lambda_{2}}{\alpha-\beta}\right) d_{q} s \\
& \quad=g_{1}\left(\frac{m \Lambda_{2}}{\alpha-\beta}\right) \int_{0}^{1}(t-q s)^{(\alpha-2)} d_{q} s=\frac{1}{\alpha-1} g_{1}\left(\frac{m(1-t)^{\alpha}}{\alpha-\beta}\right)=: \lambda_{1}, \\
& \int_{0}^{t}(t-q s)^{(\alpha-2)} g_{2}\left(\frac{m s^{\alpha-1}}{\Gamma_{q}(\alpha)}\right) d_{q} s \\
& =\left(\frac{1}{d}\right)^{1 / p}\left(\frac{\Gamma_{q}(\alpha)}{m}\right)^{\frac{1}{(\alpha-1) \kappa}}\left[\int_{0}^{\left(\frac{m}{\Gamma_{q}(\alpha)}\right) \frac{1}{\alpha-1}} g_{2}^{\kappa}\left(s^{\alpha-1}\right) d_{q} s\right]^{1 / \kappa}=: \lambda_{2},
\end{aligned}
$$


and analogously

$$
\begin{aligned}
& \int_{0}^{t}(t-q s)^{(\alpha-2)} g_{3}\left(\frac{m s^{\alpha-\beta}}{\Gamma_{q}(\alpha-\beta+1)}\right) d_{q} s \\
& \quad=\left(\frac{1}{d}\right)^{1 / p}\left(\frac{\Gamma_{q}(\alpha-\beta+1)}{m}\right)^{\frac{1}{(\alpha-\beta) \kappa}}\left[\int_{0}^{\left(\frac{m}{\Gamma_{q}(\alpha-\beta+1)}\right)^{\frac{1}{\alpha-\beta}}} g_{3}^{\kappa}\left(s^{\alpha-\beta}\right) d_{q} s\right]^{1 / \kappa} \\
& \quad=: \lambda_{3} .
\end{aligned}
$$

Note that (H1) guarantees $\lambda_{j}<\infty$ for $j=1,2$ and 3. Hence, for all $t \in \bar{J}$, we obtain

$$
\int_{0}^{t}(t-q s)^{(\alpha-2)} F(s) d_{q} s \leq \lambda,
$$

where $\lambda=\lambda_{1}+\lambda_{2}+\lambda_{3}$. Also, we have

$$
\begin{aligned}
& \int_{0}^{1} F(q s) d_{q} s \leq \frac{1}{\alpha-1} g_{1}\left(\frac{m \Lambda_{2}}{\alpha-\beta}\right)+\left(\frac{\Gamma_{q}(\alpha)}{m}\right)^{\frac{1}{\alpha-1}} \int_{0}^{\left(\frac{m}{\Gamma_{q}(\alpha)}\right)^{\frac{1}{\alpha-1}}} g_{2}\left(s^{\alpha-1}\right) d s \\
&+\left(\frac{\Gamma_{q}(\alpha-\beta+1)}{m}\right)^{\frac{1}{\alpha-\beta}} \int_{0}^{\left(\frac{m}{\Gamma_{q}(\alpha-\beta+1)}\right)^{\frac{1}{\alpha-\beta}}} g_{3}\left(s^{\alpha-\beta}\right) d_{q} s \\
&<\infty .
\end{aligned}
$$

Now, we conclude from the estimates

$$
\left\|f_{n}\right\|=\Lambda_{1}\left[\int_{0}^{1} F(q s) d_{q} s+w\left(1+\left\|f_{n}\right\|_{*}, 1+\left\|f_{n}\right\|_{*}, 1+\frac{\left\|f_{n}\right\|_{*}}{\Gamma_{q}(\beta)}\right)\|\gamma\|_{1}\right]
$$

and

$$
\left\|f_{n}^{\prime}\right\| \leq \frac{1}{\Gamma_{q}(\alpha-1)}\left[\lambda+w\left(1+\left\|f_{n}\right\|_{*}, 1+\left\|f_{n}\right\|_{*}, 1+\frac{\left\|f_{n}\right\|_{*}}{\Gamma_{q}(\beta)}\right)\left(\frac{1}{d}\right)^{1 / p}\|\gamma\|_{\kappa}\right]
$$

to the inequality

$$
\left\|f_{n}\right\|_{*} \leq M\left[\eta_{1}+\eta_{2} w\left(1+\left\|f_{n}\right\|_{*}, 1+\left\|f_{n}\right\|_{*}, 1+\frac{\left\|f_{n}\right\|_{*}}{\Gamma_{q}(\beta)}\right)\right]
$$

holding, for $n \geq 1$, where $M=\max \left\{\Lambda_{1}, \frac{1}{\Gamma_{q}(\alpha-1)}\right\}, \eta_{1}=\max \left\{\lambda, \int_{0}^{1} F(q s) d_{q} s\right\}$ and

$$
\eta_{2}=\max \left\{\|\gamma\|_{1},\left(\frac{1}{d}\right)^{1 / p}\|\gamma\|_{\kappa}\right\}
$$

Now, by condition (H1), there exists a positive constant $L$ such that

$$
M\left[\eta_{1}+\eta_{2} w\left(1+v, 1+v, 1+\frac{v}{\Gamma_{q}(\beta)}\right)\right]<v,
$$


for each $v$ higher than or equal to $L$. Now, inequality (13) gives $\left\|f_{n}\right\|_{*}<L$, for all $n$. Therefore

$$
w_{n}\left(t, f_{n}(t), f_{n}^{\prime}(t),\left({ }^{c} D_{q}^{\beta} f_{n}\right)(t)\right) \leq R(t),
$$

where $R(t)=F(t)+\gamma(t) \theta\left(1+L, 1+L, 1+\frac{L}{\Gamma_{q}(\beta)}\right)$. Note that, from condition (H1), $R$ in $L^{\kappa}(\bar{J})$. Let

$$
\tau_{n}(t)=w_{n}\left(t, f_{n}(t), f_{n}^{\prime}(t),\left({ }^{c} D_{q}^{\beta} f_{n}\right)(t)\right)
$$

and $t_{1}, t_{2} \in[0, \delta]$ such that $t_{1} \leq t_{2}$. Then

$$
\begin{aligned}
\left|f_{n}^{\prime}\left(t_{2}\right)-f_{n}^{\prime}\left(t_{1}\right)\right|= & \left(I_{q}^{\alpha-1}\right)\left|\tau_{n}\left(t_{2}\right)-\tau_{n}\left(t_{1}\right)\right| \\
\leq & \frac{1}{\Gamma_{q}(\alpha-1)}\left[\int_{0}^{t_{1}}\left(\left(t_{1}-q s\right)^{(\alpha-2)}-\left(t_{2}-q s\right)^{(\alpha-2)}\right) \tau_{n}(s) d_{q} s\right. \\
& \left.+\int_{t_{1}}^{t_{2}}\left(t_{2}-q s\right)^{(\alpha-2)} \tau_{n}(s) d_{q} s\right] \\
\leq & \frac{1}{\Gamma_{q}(\alpha-1)}\left[\int_{0}^{t_{1}}\left(\left(t_{1}-q s\right)^{(\alpha-2)}-\left(t_{2}-q s\right)^{(\alpha-2)}\right) R(s) d_{q} s\right. \\
& \left.+\int_{t_{1}}^{t_{2}}\left(t_{2}-q s\right)^{(\alpha-2)} R(s) d_{q} s\right]
\end{aligned}
$$

and so, by applying Lemma 4, we get

$$
\left|f_{n}^{\prime}\left(t_{2}\right)-f_{n}^{\prime}\left(t_{1}\right)\right| \leq \frac{\|R\|_{\kappa}}{\Gamma_{q}(\alpha-1)}\left[\left(\frac{t_{1}^{d}+\left(t_{2}-t_{1}\right)^{d}-t_{2}^{d}}{d}\right)^{1 / p}+\left(\frac{\left(t_{2}-t_{1}\right)^{d}}{d}\right)^{1 / p}\right] .
$$

As a consequence, $\left\{f_{n}^{\prime}\right\}$ is equicontinuous on $\bar{J}$. Since $\left\{f_{n}\right\}$ is bounded in $C(\bar{J})$, without less of generality, we may assume that $\left\{f_{n}\right\}$ is convergent in $C(\bar{J})$ by the Arzelà-Ascoli theorem. Let $\lim _{n \rightarrow \infty} f_{n}=f$, then passing to the limit as $n \rightarrow \infty$, we obtain $\left({ }^{c} D_{q}^{\beta} f_{n}\right)(t)=\left(I_{q}^{\alpha-1} f_{n}^{\prime}\right)(t)$ and using Eq. (7), we have

$$
\lim _{n \rightarrow \infty}\left({ }^{c} D_{q}^{\beta} f_{n}\right)(t)=\frac{1}{\Gamma_{q}(\alpha-1)} \int_{0}^{t}(t-q s)^{(-\beta)} f^{\prime}(s) d_{q} s,
$$

uniformly on $\bar{J}$. The last relation yields $\lim _{n \rightarrow \infty}\left({ }^{c} D_{q}^{\beta} f_{n}\right)(t)=\left({ }^{c} D_{q}^{\beta} f\right)(t)$ in $C(\bar{J})$. Hence,

$$
\lim _{n \rightarrow \infty} w_{n}\left(t, f_{n}(t), f_{n}^{\prime}(t),\left({ }^{c} D_{q}^{\beta} f_{n}\right)(t)\right)=w\left(t, f(t), f^{\prime}(t),\left({ }^{c} D_{q}^{\beta} f\right)(t)\right) .
$$

Since $R \in L^{\kappa}(\bar{J})$, by taking $n \rightarrow \infty$ in the equality

$$
f_{n}(t)=\int_{0}^{1} G_{q}(t, s) w_{n}\left(s, f_{n}(s), f_{n}^{\prime}(s),\left({ }^{c} D_{q}^{\beta} f_{n}\right)(s)\right) d_{q} s .
$$

By using the dominated convergence theorem for $L^{\kappa}(\bar{J})$, we get

$$
f(t)=\int_{0}^{1} G_{q}(t, s) w\left(s, f(s), f^{\prime}(s),\left({ }^{c} D_{q}^{\beta} f\right)(s)\right) d_{q} s .
$$


Consequently, $f$ is a solution of problem (1), satisfying the boundary conditions. This completes our proof.

\section{Algorithms and examples}

In this section, we give some algorithms to illustrate problem (1), in Theorems 10 and present numerical examples. Foremost, we present a simplified analysis that can be executed to calculate the value of $q$-Gamma function, $\Gamma_{q}(x)$, for input $q, x$ and different values of $n$. To this aim, we consider a pseudo-code description of the method for calculating the $q$-Gamma function of order $n$ in Algorithm 2 (for details, see the link https://en.wikipedia.org/wiki/Q-gamma_function). Now we give some examples to illustrate our results. Table 1 shows that when $q$ is constant, the $q$-Gamma function is an increasing function. Also, for smaller values of $x$, an approximate result is obtained with smaller values of $n$. It is shown by underlined rows. Table 2 shows that the $q$-Gamma function for values $q$ close to 1 is obtained with higher values of $n$ in comparison with other columns. They have been underlined in line 8 of the first column, line 17 of the second column and line 29 of third columns of Table 2. Also, Table 3 is the same as Table 2, but $x$ values increased in 3. Similarly, the $q$-Gamma function for values of $q$ close to 1 is obtained with higher values of $n$ in comparison with other columns.

Here, we provide an example to illustrate our main result.
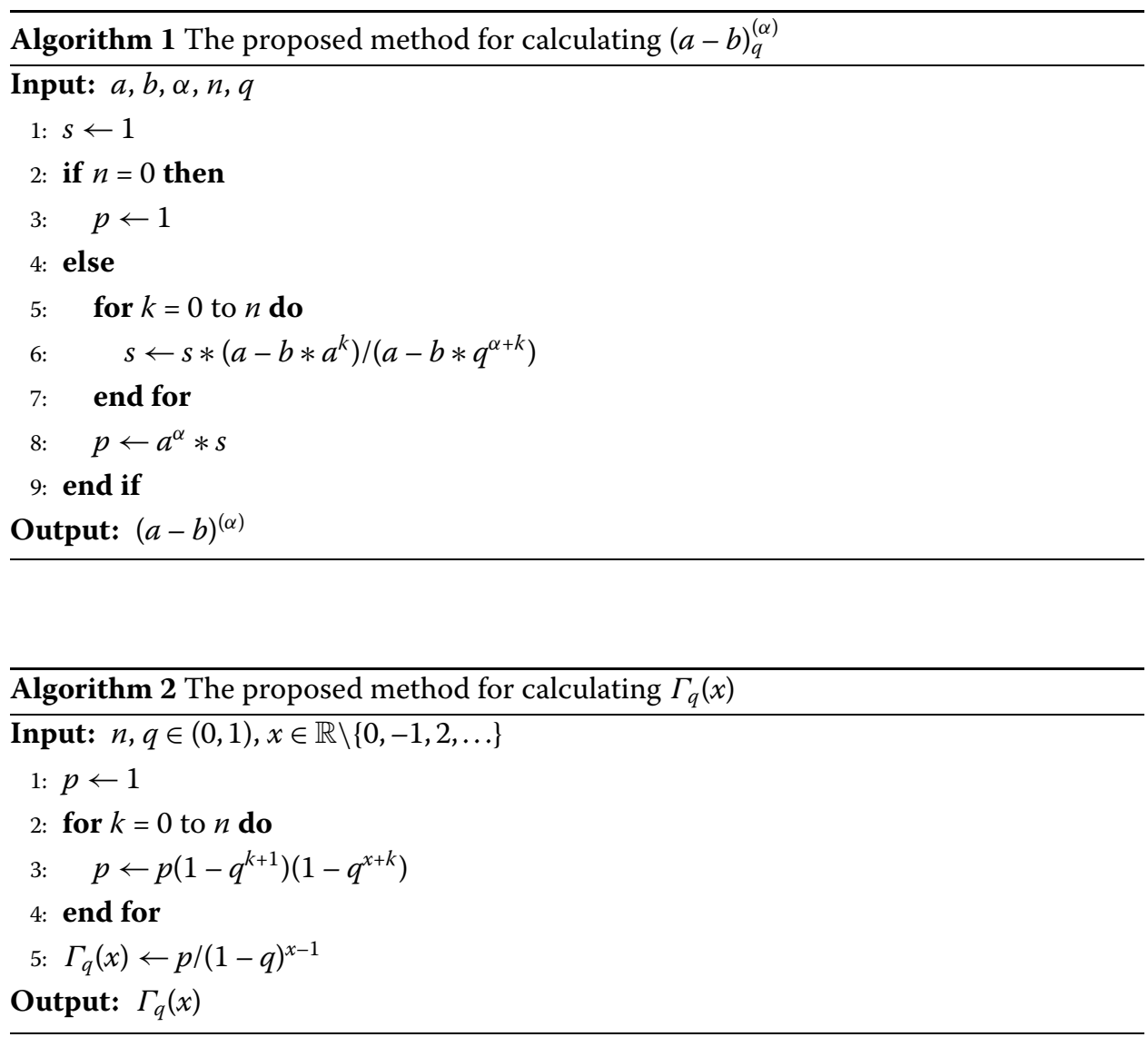
Table 1 Some numerical results for calculation of $\Gamma_{q}(x)$ with $q=\frac{1}{3}$ that is constant, $x=4.5,8.4,12.7$ and $n=1,2, \ldots, 15$ of Algorithm 2

\begin{tabular}{|c|c|c|c|c|c|c|c|}
\hline$n$ & $x=4.5$ & $x=8.4$ & $x=12.7$ & $n$ & $x=4.5$ & $x=8.4$ & $x=12.7$ \\
\hline 1 & 2.472950 & 11.909360 & 68.080769 & 9 & 2.340263 & 11.257158 & 64.351366 \\
\hline 2 & 2.383247 & 11.468397 & 65.559266 & 10 & $\overline{2.340250}$ & 11.257095 & 64.351003 \\
\hline 3 & 2.354446 & 11.326853 & 64.749894 & 11 & 2.340245 & $\overline{11.257074}$ & 64.350881 \\
\hline 4 & 2.344963 & 11.280255 & 64.483434 & 12 & 2.340244 & 11.257066 & 64.350841 \\
\hline 5 & 2.341815 & 11.264786 & 64.394980 & 13 & 2.340243 & 11.257064 & 64.350828 \\
\hline 6 & 2.340767 & 11.259636 & 64.365536 & 14 & 2.340243 & 11.257063 & 64.350823 \\
\hline 7 & 2.340418 & 11.257921 & 64.355725 & 15 & 2.340243 & 11.257063 & 64.350822 \\
\hline 8 & 2.340301 & 11.257349 & 64.352456 & & & & \\
\hline
\end{tabular}

Table 2 Some numerical results for calculation of $\Gamma_{q}(x)$ with $q=\frac{1}{3}, \frac{1}{2}, \frac{2}{3}, x=5$ and $n=1,2, \ldots, 35$ of Algorithm 2

\begin{tabular}{rlllllll}
\hline$n$ & $q=\frac{1}{3}$ & $q=\frac{1}{2}$ & $q=\frac{2}{3}$ & $n$ & $q=\frac{1}{3}$ & $q=\frac{1}{2}$ & $q=\frac{2}{3}$ \\
\hline 1 & 3.016535 & 6.291859 & 18.937427 & 18 & 2.853224 & 4.921884 & 8.476643 \\
2 & 2.906140 & 5.548726 & 14.154784 & 19 & 2.853224 & 4.921879 & 8.474597 \\
3 & 2.870699 & 5.222330 & 11.819974 & 20 & 2.853224 & 4.921877 & 8.473234 \\
4 & 2.859031 & 5.069033 & 10.537540 & 21 & 2.853224 & 4.921876 & 8.472325 \\
5 & 2.855157 & 4.994707 & 9.782069 & 22 & 2.853224 & 4.921876 & 8.471719 \\
6 & 2.853868 & 4.958107 & 9.317265 & 23 & 2.853224 & 4.921875 & 8.471315 \\
7 & 2.853438 & 4.939945 & 9.023265 & 24 & 2.853224 & 4.921875 & 8.471046 \\
8 & 2.853295 & 4.930899 & 8.833940 & 25 & 2.853224 & 4.921875 & 8.470866 \\
9 & 2.853247 & 4.926384 & 8.710584 & 26 & 2.853224 & 4.921875 & 8.470747 \\
10 & 2.853232 & 4.924129 & 8.629588 & 27 & 2.853224 & 4.921875 & 8.470667 \\
11 & 2.853226 & 4.923002 & 8.576133 & 28 & 2.853224 & 4.921875 & 8.470614 \\
12 & 2.853224 & 4.922438 & 8.540736 & 29 & 2.853224 & 4.921875 & 8.470578 \\
13 & 2.853224 & 4.922157 & 8.517243 & 30 & 2.853224 & 4.921875 & 8.470555 \\
14 & 2.853224 & 4.922016 & 8.501627 & 31 & 2.853224 & 4.921875 & 8.470539 \\
15 & 2.853224 & 4.921945 & 8.491237 & 32 & 2.853224 & 4.921875 & 8.470529 \\
16 & 2.853224 & 4.921910 & 8.484320 & 33 & 2.853224 & 4.921875 & 8.470522 \\
17 & 2.853224 & 4.921893 & 8.479713 & 34 & 2.853224 & 4.921875 & 8.470517 \\
\hline
\end{tabular}

Table 3 Some numerical results for calculation of $\Gamma_{q}(x)$ with $x=8.4, q=\frac{1}{3}, \frac{1}{2}, \frac{2}{3}$ and $n=1,2, \ldots, 40$ of Algorithm 2

\begin{tabular}{rlllllll}
\hline$n$ & $q=\frac{1}{3}$ & $q=\frac{1}{2}$ & $q=\frac{2}{3}$ & $n$ & $q=\frac{1}{3}$ & $q=\frac{1}{2}$ & $q=\frac{2}{3}$ \\
\hline 1 & 11.909360 & 63.618604 & 664.767669 & 21 & 11.257063 & 49.065390 & 260.033372 \\
2 & 11.468397 & 55.707508 & 474.800503 & 22 & 11.257063 & 49.065384 & 260.011354 \\
3 & 11.326853 & 52.245122 & 384.795341 & 23 & 11.257063 & 49.065381 & 259.996678 \\
4 & 11.280255 & 50.621828 & 336.326796 & 24 & 11.257063 & 49.065380 & 259.986893 \\
5 & 11.264786 & 49.835472 & 308.146441 & 25 & 11.257063 & 49.065379 & 259.980371 \\
6 & 11.259636 & 49.448420 & 290.958806 & 26 & 11.257063 & 49.065379 & 259.976023 \\
7 & 11.257921 & 49.256401 & 280.150029 & 27 & 11.257063 & 49.065379 & 259.973124 \\
8 & 11.257349 & 49.160766 & 273.216364 & 28 & 11.257063 & 49.065378 & 259.971192 \\
9 & 11.257158 & 49.113041 & 268.710272 & 29 & 11.257063 & 49.065378 & 259.969903 \\
10 & 11.257095 & 49.089202 & 265.756606 & 30 & 11.257063 & 49.065378 & 259.969044 \\
11 & 11.257074 & 49.077288 & 263.809514 & 31 & 11.257063 & 49.065378 & 259.968472 \\
12 & 11.257066 & 49.071333 & 262.521127 & 32 & 11.257063 & 49.065378 & 259.968090 \\
13 & 11.257064 & 49.068355 & 261.666471 & 33 & 11.257063 & 49.065378 & 259.967836 \\
14 & 11.257063 & 49.066867 & 261.098587 & 34 & 11.257063 & 49.065378 & 259.967666 \\
15 & 11.257063 & 49.066123 & 260.720833 & 35 & 11.257063 & 49.065378 & 259.967553 \\
16 & 11.257063 & 49.065751 & 260.469369 & 36 & 11.257063 & 49.065378 & 259.967478 \\
17 & 11.257063 & 49.065564 & 260.301890 & 37 & 11.257063 & 49.065378 & 259.967427 \\
18 & 11.257063 & 49.065471 & 260.190310 & 38 & 11.257063 & 49.065378 & $\underline{259.967394}$ \\
19 & 11.257063 & 49.065425 & 260.115957 & 39 & 11.257063 & 49.065378 & 259.967371 \\
20 & 11.257063 & 49.065402 & 260.066402 & 40 & 11.257063 & 49.065378 & 259.967357 \\
\hline
\end{tabular}


Example 1 Let $\bar{J}=[0,1], \tau_{1}$ and $\tau_{2}$ belongs to $L^{\kappa}(\bar{J})$ and $\tau_{1}(t)$ higher than or equal to positive real number $m$ for all $t \in \bar{J}$. Also, let

$$
\begin{aligned}
w\left(t, x_{1}, x_{2}, x_{3}\right)= & \tau_{1}(t)+\frac{1}{x_{1}^{2 / 5}-r}+\frac{1}{x_{2}^{1 / 4}}+\frac{1}{x_{3}^{1 / 4}} \\
& +\left|\tau_{2}(t)\right|\left(x_{1}^{2 / 5}+x_{2}^{1 / 4}+x_{3}^{1 / 4}\right),
\end{aligned}
$$

on $\bar{J} \times A$ with $A=[0, \infty) \times[0, \infty) \times[0, \infty), g_{1}(u)=\frac{1}{u^{2 / 5}-r}$ whenever $u^{2 / 5} \geq r$ and $g_{1}(u)=0$ whenever $u^{2 / 5}<r, g_{2}(u)=\frac{1}{u^{1 / 4}}, g_{3}(u)=\frac{1}{u^{1 / 4}}$,

$$
w\left(x_{1}, x_{2}, x_{3}\right)=x_{1}^{2 / 5}+x_{2}^{1 / 4}+x_{3}^{1 / 4}+1
$$

and $\gamma(t)=\tau_{1}(t)+\left|\tau_{2}(t)\right|$, where $r=(a f(1))^{2 / 5}$. Since $w$ satisfies conditions (H1) and (H2), Theorem 10 guarantees that problem (1) has a positive solution.

Example 2 In this example, we choose a problem similar to (1),

$$
\left\{\begin{array}{l}
{ }^{c} D_{q}^{9 / 4} f(t)=t+1+\frac{1}{(f(t))^{2 / 5}-\lambda}+\frac{1}{\left(f^{\prime}(t)\right)^{1 / 4}}+\frac{1}{\left[\left({ }^{c} D_{q}^{1 / 4} f\right)(t)\right]^{1 / 4}} \\
\quad+2\left(f(t)^{2 / 5}+f^{\prime}(t)^{1 / 4}+\left[\left({ }^{c} D_{q}^{1 / 4} f\right)(t)\right]^{1 / 4}+1\right), \\
f(0)=\frac{1}{4} f(1) \\
f^{\prime}(0)=\frac{1}{3}\left({ }^{c} D_{q}^{1 / 4} f\right)(1), \\
f^{\prime \prime}(0)=f^{\prime \prime \prime}(0)=\cdots=f^{(n-1)}(0)=0,
\end{array}\right.
$$

where $\lambda=\left(\frac{1}{4} f(1)\right)^{1 / 3}$. here $\alpha=\frac{9}{4} \in(2,3)$, with $n=3, \beta=\frac{1}{4} \in(0,1), c_{1}=\frac{1}{4} \in(0,1), c_{2}=\frac{1}{3} \in$ $\left(0, \Gamma_{q}\left(\frac{7}{4}\right)\right)$ for all $q \in(0,1)$ and $\kappa\left(\frac{9}{4}-1\right)=\frac{4}{5}>1$. Then

$$
\begin{aligned}
w\left(t, f(t), f^{\prime}(t),\left({ }^{c} D_{q}^{1 / 4} f\right)(t)\right)= & t+1+\frac{1}{f(t)^{1 / 3}-\lambda}+\frac{1}{f^{\prime}(t)^{1 / 4}} \\
& +\frac{1}{\left[\left({ }^{c} D_{q}^{1 / 4} f\right)(t)\right]^{1 / 4}} \\
& +2\left(f(t)^{1 / 3}+r^{\prime}(t)^{1 / 4}+\left[{ }^{c} D_{q}^{1 / 4} f(t)\right]^{1 / 4}+1\right),
\end{aligned}
$$

and $w$ may be singular at $t=0$ and satisfies conditions (H1) and (H2), for $g_{1}(h)=\frac{1}{h^{1 / 3}-\lambda}$ whenever $h^{1 / 3}-k \geq 0$ and $g_{1}(h)=0$ whenever $h^{1 / 3}-\lambda<0, g_{2}(h)=\frac{1}{h^{1 / 4}}, g_{3}(h)=\frac{1}{h^{1 / 4}}$,

$$
w\left(x_{1}, x_{2}, x_{3}\right)=x_{1}^{1 / 3}+x_{2}^{1 / 4}+x_{3}^{1 / 4}+1
$$

and $\tau_{1}(t)=t+1>1=m, \tau_{2}(t)=2$ and $\gamma(t)=\tau_{1}(t)+\left|\tau_{2}(t)\right|$, Theorem 10 guarantees that problem (1) has a positive solution. Now, we investigate the computational complexity of Example 2 of Algorithm 6 and 7. Note that $n$ in Algorithms 6 and 7 is used for calculating $\Gamma_{q}(x)$. Tables 4, 5 and 6 show the values of $\Lambda_{1}$ and $\Lambda_{2}$ for $q=\frac{1}{3}, \frac{1}{2}$ and $\frac{3}{4}$, respectively, an approximate result is obtained with less than four decimal places indicated by underlining. 


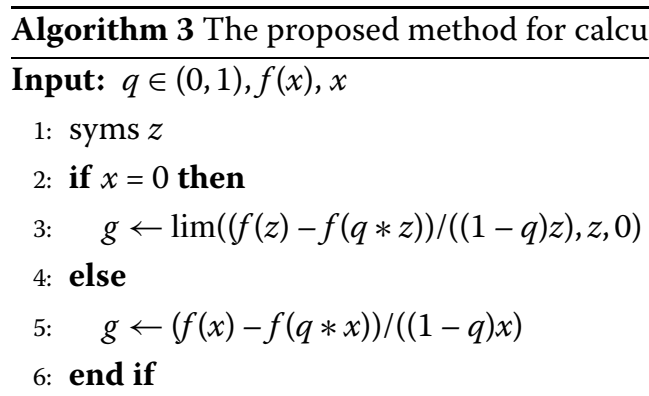

Output: $\left(D_{q} f\right)(x)$

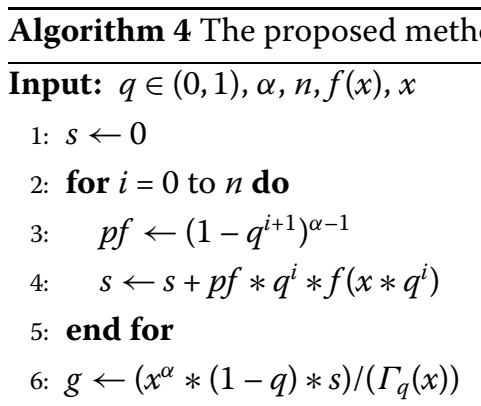

Output: $\left(I_{q}^{\alpha} f\right)(x)$

Algorithm 5 The proposed method for calculating $\int_{a}^{b} f(r) d_{q} r$

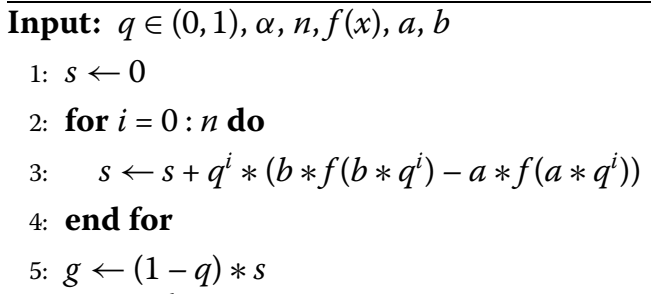

Output: $\int_{a}^{b} f(r) d_{q} r$

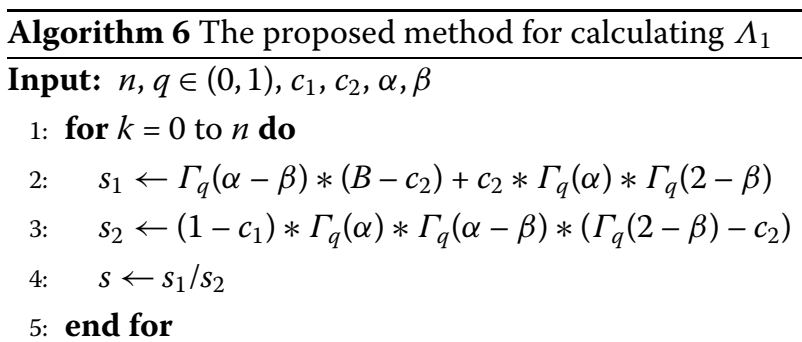

Output: $\Lambda_{1}=s$

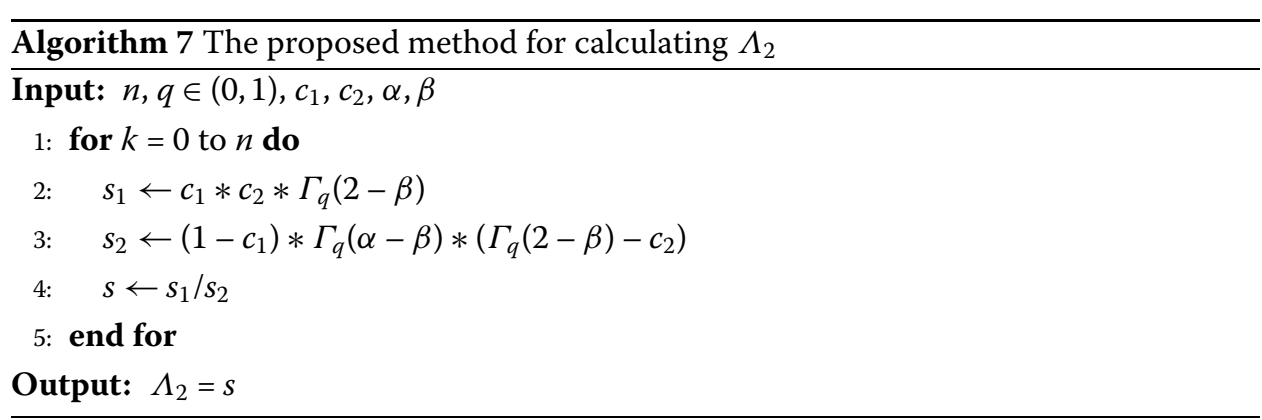


Table 4 Some numerical results for calculation of $\Lambda_{1}$ and $\Lambda_{2}$ with $q=\frac{1}{3}$ and $n=1,2, \ldots, 12$ of Example 2

\begin{tabular}{rlllll}
\hline$n$ & $\Gamma_{q}(2-\beta)$ & $\Gamma_{q}(\alpha-\beta)$ & $\Gamma_{q}(\alpha)$ & $\Lambda_{1}$ & $\Lambda_{2}$ \\
\hline 1 & 0.988977 & 1.038462 & 1.105539 & -1.110973 & -0.871523 \\
2 & 0.968078 & 1.0125 & 1.074674 & -1.146096 & -0.90379 \\
3 & 0.961333 & 1.004132 & 1.064736 & -1.15789 & -0.914692 \\
4 & 0.959108 & 1.001374 & 1.061461 & -1.16183 & -0.918342 \\
5 & 0.958369 & 1.000457 & 1.060373 & -1.163145 & -0.919561 \\
6 & 0.958123 & 1.000152 & 1.060011 & -1.163583 & -0.919967 \\
7 & 0.958041 & 1.000051 & 1.059891 & -1.16373 & -0.920103 \\
8 & 0.958014 & 1.000017 & 1.05985 & -1.163778 & -0.920148 \\
9 & 0.958005 & 1.000006 & 1.059837 & -1.163794 & -0.920163 \\
10 & 0.958002 & 1.000002 & 1.059832 & -1.1638 & -0.920168 \\
11 & 0.958001 & 1.000001 & 1.059831 & -1.163802 & -0.92017 \\
12 & 0.958 & 1 & 1.05983 & -1.163802 & -0.92017 \\
\hline
\end{tabular}

Table 5 Some numerical results for calculation of $\Lambda_{1}$ and $\Lambda_{2}$ with $q=\frac{1}{2}$ and $n=1,2, \ldots, 19$ of Example 2

\begin{tabular}{rlllll}
\hline$n$ & $\Gamma_{q}(2-\beta)$ & $\Gamma_{q}(\alpha-\beta)$ & $\Gamma_{q}(\alpha)$ & $\Lambda_{1}$ & $\Lambda_{2}$ \\
\hline 1 & 1.05421 & 1.142857 & 1.261962 & -0.97516 & -0.76776 \\
2 & 0.996499 & 1.066667 & 1.165469 & -1.062079 & -0.845235 \\
3 & 0.970276 & 1.032258 & 1.122114 & -1.106468 & -0.885437 \\
4 & 0.957751 & 1.015873 & 1.101521 & -1.128899 & -0.905919 \\
5 & 0.951628 & 1.007874 & 1.09148 & -1.140174 & -0.916256 \\
6 & 0.9486 & 1.003922 & 1.086522 & -1.145827 & -0.921449 \\
7 & 0.947094 & 1.001957 & 1.084058 & -1.148657 & -0.924052 \\
8 & 0.946343 & 1.000978 & 1.08283 & -1.150073 & -0.925354 \\
9 & 0.945968 & 1.000489 & 1.082217 & -1.150782 & -0.926006 \\
10 & 0.945781 & 1.000244 & 1.081911 & -1.151136 & -0.926332 \\
11 & 0.945687 & 1.000122 & 1.081758 & -1.151313 & -0.926495 \\
12 & 0.945641 & 1.000061 & 1.081681 & -1.151401 & -0.926577 \\
13 & 0.945617 & 1.000031 & 1.081643 & -1.151446 & -0.926618 \\
14 & 0.945606 & 1.000015 & 1.081624 & -1.151468 & -0.926638 \\
15 & 0.9456 & 1.000008 & 1.081614 & -1.151479 & -0.926648 \\
16 & 0.945597 & 1.000004 & 1.081609 & -1.151485 & -0.926653 \\
17 & 0.945595 & 1.000002 & 1.081607 & -1.151487 & -0.926656 \\
18 & 0.945595 & 1.000001 & 1.081606 & -1.151489 & -0.926657 \\
19 & 0.945594 & 1.000000 & 1.081605 & -1.151489 & -0.926658 \\
\hline
\end{tabular}


Table 6 Some numerical results for calculation of $\Lambda_{1}$ and $\Lambda_{2}$ with $q=\frac{3}{4}$ and $n=1,2, \ldots, 30$ of Example 2

\begin{tabular}{rlllll}
\hline$n$ & $\Gamma_{q}(2-\beta)$ & $\Gamma_{q}(\alpha-\beta)$ & $\Gamma_{q}(\alpha)$ & $\Lambda_{1}$ & $\Lambda_{2}$ \\
\hline 2 & 1.253179 & 1.462857 & 1.751525 & -0.705095 & -0.558789 \\
3 & 1.149887 & 1.31114 & 1.536689 & -0.807011 & -0.644426 \\
4 & 1.084407 & 1.216513 & 1.40468 & -0.886016 & -0.712105 \\
5 & 1.040678 & 1.154047 & 1.318456 & -0.946732 & -0.764915 \\
6 & 1.010469 & 1.111251 & 1.259837 & -0.993102 & -0.805725 \\
7 & 0.989113 & 1.08118 & 1.218879 & -1.028354 & -0.83703 \\
8 & 0.973772 & 1.059674 & 1.189708 & -1.055062 & -0.860912 \\
9 & 0.962624 & 1.044098 & 1.168644 & -1.075245 & -0.879054 \\
10 & 0.954455 & 1.032713 & 1.153282 & -1.090469 & -0.892792 \\
11 & 0.948434 & 1.024335 & 1.141999 & -1.101936 & -0.903171 \\
12 & 0.943976 & 1.018141 & 1.133666 & -1.110563 & -0.910997 \\
13 & 0.940664 & 1.013544 & 1.127488 & -1.117049 & -0.916891 \\
14 & 0.938198 & 1.010124 & 1.122894 & -1.121923 & -0.921325 \\
15 & 0.936358 & 1.007574 & 1.119471 & -1.125583 & -0.924658 \\
16 & 0.934984 & 1.00567 & 1.116915 & -1.12833 & -0.927162 \\
17 & 0.933956 & 1.004246 & 1.115006 & -1.130393 & -0.929043 \\
18 & 0.933187 & 1.003181 & 1.113578 & -1.13194 & -0.930455 \\
19 & 0.932611 & 1.002384 & 1.112509 & -1.133102 & -0.931514 \\
20 & 0.93218 & 1.001787 & 1.111708 & -1.133973 & -0.932309 \\
21 & 0.931857 & 1.00134 & 1.111109 & -1.134627 & -0.932906 \\
22 & 0.931615 & 1.001004 & 1.110659 & -1.135117 & -0.933354 \\
23 & 0.931433 & 1.000753 & 1.110322 & -1.135485 & -0.933689 \\
24 & 0.931297 & 1.000565 & 1.11007 & -1.13576 & -0.933941 \\
25 & 0.931195 & 1.000423 & 1.10988 & -1.135967 & -0.93413 \\
26 & 0.931118 & 1.000318 & 1.109738 & -1.136122 & -0.934272 \\
27 & 0.931061 & 1.000238 & 1.109632 & -1.136239 & -0.934378 \\
28 & 0.931018 & 1.000179 & 1.109552 & -1.136326 & -0.934458 \\
29 & 0.930986 & 1.000134 & 1.109492 & -1.136392 & -0.934518 \\
30 & 0.930961 & 1.0001 & 1.109447 & -1.136441 & -0.934562 \\
\hline & & & & &
\end{tabular}

\section{Funding}

Not applicable

Availability of data and materials

Not applicable.

Ethics approval and consent to participate

Not applicable.

\section{Competing interests}

The authors declare that they have no competing interests.

Consent for publication

Not applicable.

Authors' contributions

All authors contributed equally and significantly in this manuscript and they read and approved the final manuscript.

\section{Author details}

'College of Mathematics, Changchun Normal University, Jilin, P.R. China. ${ }^{2}$ Department of Mathematics, Bu-Ali Sina University, Hamedan, Iran.

\section{Publisher's Note}

Springer Nature remains neutral with regard to jurisdictional claims in published maps and institutional affiliations.

Received: 22 November 2019 Accepted: 29 December 2019 Published online: 07 January 2020

\section{References}

1. Jackson, F.H.: q-difference equations. Am. J. Math. 32, 305-314 (1910). https://doi.org/10.2307/2370183

2. Stanković, M.S., Rajković, P.M., Marinković, S.D.: On -fractional derivatives of Riemann-Liouville and Caputo type (2009) arXiv:0909.0387 
3. Rajković, P.M., Marinković, S.D., Stanković, M.S.: Fractional integrals and derivatives in q-calculus. Appl. Anal. Discrete Math. 1, 311-323 (2007)

4. Annaby, M.H., Mansour, Z.S.: q-Fractional Calculus and Equations. Springer, Cambridge (2012). https://doi.org/10.1007/978-3-642-30898-7

5. Kilbas, A.A., Srivastava, H.M., Trujillo, J.J.: Theory and Applications of Fractional Differential Equations, North-Holland Mathematics Studies. Elsevier, Amsterdam (2006)

6. Kac, V., Cheung, P.: Quantum Calculus. Universitext. Springer, New York (2002). https://doi.org/10.1007/978-1-4613-0071-7-1

7. Goodrich, C., Peterson, A.C.: Discrete Fractional Calculus. Springer, Switzerland (2015). https://doi.org/10.1007/978-3-319-25562-0

8. Podlubny, I.: Fractional Differential Equations. Academic Press, San Diego (1999)

9. Shi, K., Wang, J., Tang, Y., Zhong, S.: Reliable asynchronous sampled-data filtering of t-s fuzzy uncertain delayed neural networks with stochastic switched topologies. Fuzzy Sets Syst. 381(15), 1-25 (2020). https://doi.org/10.1016/j.fss.2018.11.017

10. Ahmad, B., Nieto, J.J., Alsaedi, A., Al-Hutami, H.: Existence of solutions for nonlinear fractional q-difference integral equations with two fractional orders and nonlocal four-point boundary conditions. J. Franklin Inst. 351, 2890-2909 (2014). https://doi.org/10.1016/j.jfranklin.2014.01.020

11. Miller, K.S., Ross, B.: An Introduction to Fractional Calculus and Fractional Differential Equations. Wiley, New York (1993)

12. Atici, F., Eloe, P.W.: Fractional q-calculus on a time scale. J. Nonlinear Math. Phys. 14(3), 341-352 (2007). https://doi.org/10.2991/jnmp.2007.14.3.4

13. Alsaedi, A., Baleanu, D., Etemad, S., Rezapour, S.: On coupled systems of time-fractional differential problems by using a new fractional derivative. J. Funct. Spaces 2016, 4626940 (2016). https://doi.org/10.1155/2016/4626940

14. Agarwal, R.P., Baleanu, D., Hedayati, V., Rezapour, S.: Two fractional derivative inclusion problems via integral boundary condition. Appl. Math. Comput. 257, 205-212 (2015). https://doi.org/10.1016/j.amc.2014.10.082

15. Baleanu, D., Rezapour, S., Mohammadi, H.: Some existence results on nonlinear fractional differential equations. Philos. Trans. R. Soc. A, Math. Phys. Eng. Sci. 371, 7 (2013). https://doi.org/10.1098/rsta.2012.0144

16. Baleanu, D., Mousalou, A., Rezapour, S.: A new method for investigating approximate solutions of some fractional integro-differential equations involving the Caputo-Fabrizio derivative. Adv. Differ. Equ. 2017, 51 (2017). https://doi.org/10.1186/s13662-017-1088-3

17. Baleanu, D., Mousalou, A., Rezapour, S.: On the existence of solutions for some infinite coefficient-symmetric Caputo-Fabrizio fractional integro-differential equations. Bound. Value Probl. 2017, 145 (2017) https://doi.org/10.1186/s13661-017-0867-9

18. Baleanu, D., Mousalou, A., Rezapour, S.: The extended fractional Caputo-Fabrizio derivative of order $0 \leq \sigma<1$ on $c_{\mathbb{R}}[0,1]$ and the existence of solutions for two higher-order series-type differential equations. Adv. Differ. Equ. 2018 255 (2018). https://doi.org/10.1186/s13662-018-1696-6

19. Akbari Kojabad, E., Rezapour, S.: Approximate solutions of a sum-type fractional integro-differential equation by using Chebyshev and Legendre polynomials. Adv. Differ. Equ. 2017, 351 (2017). https://doi.org/10.1186/s13662-017-1404-y

20. Melike Aydogan, S., Baleanu, D., Mousalou, A., Rezapour, S.: On approximate solutions for two higher-order Caputo-Fabrizio fractional integro-differential equations. Adv. Differ. Equ. 2017, 221 (2017). https://doi.org/10.1186/s13662-017-1258-3

21. Melike Aydogan, S., Baleanu, D., Mousalou, A., Rezapour, S.: On high order fractional integro-differential equations including the Caputo-Fabrizio derivative. Bound. Value Probl. 2018, 90 (2018). https://doi.org/10.1186/s13661-018-1008-9

22. Baleanu, D., Rezapour, S., Saberpour, Z:: On fractional integro-differential inclusions via the extended fractional Caputo-Fabrizio derivation. Bound. Value Probl. 2019, 79 (2019). https://doi.org/10.1186/s13661-019-1194-0

23. Baleanu, D., Ghafarnezhad, K., Rezapour, S., Shabibi, M.: On the existence of solutions of a three steps crisis integro-differential equation. Adv. Differ. Equ. 2018, 135 (2018). https://doi.org/10.1186/s13662-018-1583-1

24. Baleanu, D., Ghafarnezhad, K., Rezapour, S.: On a three step crisis integro-differential equation. Adv. Differ. Equ. 2019 153 (2019). https://doi.org/10.1186/s13662-019-2088-2

25. Baleanu, D., Etemad, S., Pourrazi, S., Rezapour, S.: On the new fractional hybrid boundary value problems with three-point integral hybrid conditions. Adv. Differ. Equ. 2019, 473 (2019). https://doi.org/10.1186/s13662-019-2088-2

26. Samei, M.E., Hedayati, V., Rezapour, S.: Existence results for a fraction hybrid differential inclusion with Caputo-Hadamard type fractional derivative. Adv. Differ. Equ. 2019, 163 (2019). https://doi.org/10.1186/s13662-019-2090-8

27. Hedayati, V., Samei, M.E.: Positive solutions of fractional differential equation with two pieces in chain interval and simultaneous Dirichlet boundary conditions. Bound. Value Probl. 2019, 163 (2019). https://doi.org/10.1186/s13661-019-1251-8

28. Al-Omari, S.K.Q., Baleanu, D., Purohit, S.D.: Some results for Laplace-type integral operator in quantum calculus. Adv. Differ. Equ. 2018, 124 (2018). https://doi.org/10.1186/s13662-018-1567-1

29. Jarad, F., Abdeljawad, T., Gündoğdu, E., Baleanu, D.: On the Mittag-Leffler stability of $q$-fractional nonlinear dynamical systems. Proc. Rom. Acad., Ser. A: Math. Phys. Tech. Sci. Inf. Sci. 12(4), 309-314 (2011)

30. Jarad, F., Abdeljawad, T., Baleanu, D.: Stability of q-fractional non-autonomous systems. Nonlinear Anal., Real World Appl. 14(1), 780-784 (2013). https://doi.org/10.1016/j.nonrwa.2012.08.001

31. Abdeljawad, T., Benli, B., Baleanu, D.: A generalized $q$-Mittag-Leffler function by $q$-Captuo fractional linear equations. Abstr. Appl. Anal. 2012, 11 (2012). https://doi.org/10.1155/2012/546062

32. Samei, M.E., Khalilzadeh Ranjbar, G.: Some theorems of existence of solutions for fractional hybrid $q$-difference inclusion. J. Adv. Math. Stud. 12(1), 63-76 (2019)

33. Kalvandi, V., Samei, M.E.: New stability results for a sum-type fractional $q$-integro-differential equation. J. Adv. Math. Stud. 12(2), 201-209 (2019)

34. Ahmad, B., Ntouyas, S.K., Purnaras, I.K.: Existence results for nonlocal boundary value problems of nonlinear fractional q-difference equations. Adv. Differ. Equ. 2012, 140 (2012). https://doi.org/10.1186/1687-1847-2012-140 
35. Ferreira, R.A.C.: Nontrivials solutions for fractional $q$-difference boundary value problems. Electron. J. Qual. Theory Differ. Equ. 2010, 70 (2010)

36. Samei, M.E., Ranjbar, G.K., Hedayati, V.: Existence of solutions for equations and inclusions of multi-term fractional q-integro-differential with non-separated and initial boundary conditions. J. Inequal. Appl. 2019, 273 (2019). https://doi.org/10.1186/s13660-019-2224-2

37. Trjitzinsky, W.J.: Analytic theory of linear q-difference equations. Acta Math. 61, 1-38 (1933). https://doi.org/10.1007/BF02547785

38. Zhao, Y., Chen, H., Zhang, Q.: Existence results for fractional $q$-difference equations with nonlocal $q$-integral boundary conditions. Adv. Differ. Equ. 2013, 48 (2013). https://doi.org/10.1186/1687-1847-2013-48

39. Jackson, F.H.: On q-definite integrals. Q. J. Pure Appl. Math. 41, 193-203 (1910). https://doi.org/10.1017/S0080456800002751

40. Al-Salam, W.A.: Some fractional q-integrals and q-derivatives. Proc. Edinb. Math. Soc. 15, 135-140 (1966-1967). https://doi.org/10.1017/S0013091500011469

41. Agarwal, R.P.: Certain fractional q-integrals and q-derivatives. Proc. Camb. Philos. Soc. 66, 365-370 (1969). https://doi.org/10.1017/S0305004100045060

42. Ahmad, B., Ntouyas, S.K., Tariboon, J.: Quantum Calculus. New Concepts, Impulsive IVPs and BVPs, Inequalities. Trends in Abstract and Applied Analysis, vol. 4. World Scientific, Hackensack (2016). https://doi.org/10.1142/10075

43. Ahmad, B., Ntouyas, S.K.: Existence of solutions for nonlinear fractional q-difference inclusions with nonlocal robin (separated) conditions. Mediterr. J. Math. 10, 133-1351 (2013). https://doi.org/10.1007/s00009-013-0258-0

44. Hilfer, R.: Applications of Fractional Calculus in Physics. World Scientific, Germany (2000). https://doi.org/10.1142/3779

45. Rezapour, S., Shabibi, M.: A singular fractional differential equation with Riemann-Liouville integral boundary condition. J. Adv. Math. Stud. 8(1), 80-88 (2015)

46. Ntouyas, S.K., Samei, M.E.: Existence and uniqueness of solutions for multi-term fractional $q$-integro-differential equations via quantum calculus. Adv. Differ. Equ. 2019, 475 (2019). https://doi.org/10.1 186/s13662-019-2414-8

47. Shabibi, M., Rezapour, S., Vaezpour, S.M.: A singular fractional integro-differential equation. UPB Sci. Bull., Ser. A 79(1), 109-118 (2017)

48. Ntouyas, S.K., Etemad, S.: On the existence of solutions for fractional differential inclusions with sum and integral boundary conditions. Appl. Math. Comput. 266, 235-243 (2017). https://doi.org/10.1016/j.amc.2015.05.036

49. Samei, M.E., Khalilzadeh Ranjbar, G., Hedayati, V.: Existence of solutions for a class of Caputo fractional $q$-difference inclusion on multifunctions by computational results. Kragujev. J. Math. 45(4), 543-570 (2021)

50. Baleanu, D., Mohammadi, H., Rezapour, S.: The existence of solutions for a nonlinear mixed problem of singular fractional differential equations. Adv. Differ. Equ. 2013, 359 (2013). https://doi.org/10.1186/1687-1847-2013-359

51. Jiang, M., Zhong, S.: Existence of solutions for nonlinear fractional q-difference equations with Riemann-Liouville type q-derivatives. J. Appl. Math. Comput. 47(1-2), 429-459 (2015). https://doi.org/10.1007/s12190-014-0784-3

52. Abdeljawad, T., Alzabut, J., Baleanu, D.: A generalized $q$-fractional Gronwall inequality and its applications to non-linear delay 9 -fractional difference systems. J. Inequal. Appl. 2016, 240 (2016). https://doi.org/10.1186/s13660-016-1181-2

53. Zhou, H., Alzabut, J., Yang, L.: On fractional Langevin differential equations with anti-periodic boundary conditions. Eur. Phys. J. Spec. Top. 226, 3577-3590 (2017). https://doi.org/10.1140/epjst/e2018-00082-0

54. Rezapour, S., Hedayati, V.: On a Caputo fractional differential inclusion with integral boundary condition for convex-compact and nonconvex-compact valued multifunctions. Kragujev. J. Math. 41(1), 143-158 (2017). https://doi.org/10.5937/KgJMath1701143R

55. El-Shahed, M., Al-Askar, F.M.: Positive solutions for boundary value problem of nonlinear fractional $q$-difference equation. International Scholarly Research Network, ISRN Mathematical Analysis 2011, 385459 (2011). https://doi.org/10.5402/2011/385459

56. Etemad, S., Ettefagh, M., Rezapour, S.: On the existence of solutions for nonlinear fractional q-difference equations with q-integral boundary conditions. J. Adv. Math. Stud. 8, 265-285 (2015)

57. Adams, C.R.: The general theory of a class of linear partial $q$-difference equations. Trans. Am. Math. Soc. 26, 283-312 (1924)

58. Gauchman, H.: Integral inequalities in q-calculus. Comput. Math. Appl. 47(2-3), 281-300 (2004). https://doi.org/10.1016/S0898-1221(04)90025-9

59. Ahmad, B., Etemad, S., Ettefagh, M., Rezapour, S.: On the existence of solutions for fractional q-difference inclusions with $q$-antiperiodic boundary conditions. Bull. Math. Soc. Sci. Math. Roum. 59(107) (2), 119-134 (2016).

60. Agarwal, R.P., O'Regan, D., Staněk, S.: The existence of solutions for a nonlinear mixed problem of singular fractional differential equations. Math. Nachr. 285(1), 27-41 (2012). https://doi.org/10.1002/mana.201000043

61. Baleanu, D., Mohammadi, H., Rezapour, S.: Positive solutions of a boundary value problem for nonlinear fractional differential equations. Abstr. Appl. Anal. 2012, 837437 (2012). https://doi.org/10.1155/2012/837437

62. Krasnosel'skij, M.A.: Positive Solutions of Operator Equations. Noordhoff, Groningen (1964) 